\title{
Fracture mechanics of rate-and-state faults and fluid injection induced slip*
}

\author{
Dmitry I. Garagash ${ }^{\dagger}$
}

\begin{abstract}
Propagation of a slip transient on a fault with rate-and-state dependent friction resembles a fracture which near tip region is characterized by large departure of the slip velocity and fault strength from the steady-state sliding. We develop a near tip solution to describe this unsteady dynamics, and obtain the fracture energy $G_{c}$, dissipated in overcoming strength-excursion away from steady-state, as a function of the rupture velocity $v_{r}$. This opens a possibility to model slip transients on rate-state faults as singular cracks characterized by approximately steady-state frictional resistance in the fracture bulk, and by a stress singularity with the intensity defined by $G_{c}\left(v_{r}\right)$ at the crack tip. In pursuing this route, we develop and use an analytical equation of motion to study 1D slip driven by a combination of uniform background stress and a localized perturbation of the fault strength with the net Coulomb force $\Delta T$. In the context of fluid injection, $\Delta T$ is a proxy for the injection volume $V_{\text {inj. }}$. We then show that, for ongoing fluid injection, the maximum propagation speed of the transient induced on a frictionally-stable fault is bounded by a value $\propto V_{\text {inj }}(t)$, and, for stopped injection, the maximum slip run-out distance is $\propto V_{\text {inj,total }}^{2}$.

Keywords: rate-and-state dependent friction, fracture mechanics, rupture fronts, equation of motion, injection-induced slip
\end{abstract}

\section{Introduction}

Rate and state dependent friction model has been widely used to describe slip behavior on the nature faults [1] and material interfaces [2]. Emerging from rock mechanics experiments [3, 4, 5], this modeling framework embodies two fundamental mechanisms governing the resistance of an interface to sliding, namely, dependence of friction on the rate of sliding and on the continually accrued slip, the latter pacing evolution of the 'state' of the interface in response to changes in slip rate. Despite the framework's limitations when applied to high (seismic) slip velocities (when the shear-heating-driven thermal pressurization of pore fluid, microscopic flash heating of frictional asperities, and possibly macroscopic melting of the fault gouge $[6,7,8]$ can dramatically alter the fault strength), the adoption of the rate-state friction in the numerical and analytical models over the last couple decades has allowed to study a wide spectra of fault slip. These include, on one hand, studies of the earthquake nucleation process $[9,10,11]$, seismic rupture styles and their transitions $[12,13,14]$, and earthquake cycle [15, 16], and, on the other, studies of slow slip transients which may arise as the result of a 'failed' earthquake instability on a frictionally-unstable (rate-weakening) fault $[17,18]$, or on presumably frictionally-stable (rate-strengthening) parts of

* Submitted for consideration by Philosophical Transactions of Royal Society A (July 5, 2020)
${ }^{\dagger}$ Dalhousie University, Dept. Civil Resource Eng., Halifax, Canada. E-mail: garagash@dal.ca 
the fault in response to the loading from a near-by earthquake [19], or due to the fault strength reduction enacted by fluid injection $[20,21]$.

A wealth of numerical simulations of slip on rate-state faults and laboratory observations of sliding on frictional interfaces ([22] and references therein) show that transient propagation of slip events bear resemblance to fracture, characterized by the leading edge well-defined by locally very large but finite slip-rate gradient (in contrast to 'pure' fracture with its infinite displacement discontinuity gradient at the leading edge). This fracture-like behavior is not limited to seismic ruptures, but rather occurs across the spectra of slip events from slow to fast. The fracture-like slow slip transients are illustrated on Figure 1 for two scenarios of fluid injection in the vicinity of a frictionally-stable (rate-strengthening) fault. The first corresponds to a fixed-volume injection into a hydraulically sealed reservoir layer abated by a fault (with no fluid leakage along the fault and out of the reservoir layer allowed), resulting in a fixed pore pressure increase $\Delta p=$ const along the fault segment in contact with the reservoir (e.g. [23]). The second corresponds to a continuing, fixedvolume-rate injection directly into the permeable fault damage zone, with ensuing pore pressure diffusion along the fault, $\Delta p=\Delta p(z, t)$, (e.g. [24]). The generated slow slip transients spread symmetrically along the 1D fault (implying fluid injection over a long line source (horizontal wellbore segment) parallel to the fault in the out-of-plane direction, see inset of Figure $\left.1 \mathrm{a}, \mathrm{a}^{\prime}\right)$ and have decelerating / approximately steady long-term dynamics in the fixed-volume / fixed-volumerate case.

Notwithstanding the diverse dynamics of slip, the transients have a well-defined leading edge (fracture tip) characterized by very large slip-rate gradient (Figure 1c, $\mathrm{c}^{\prime}$ ) and associated with the maximum value of the friction departure $\Delta f$ from its instantaneous steady-state value $\left(\mathrm{b}, \mathrm{b}^{\prime}\right)$. Snapshots of the $\Delta f$-distribution along the fault allow to identify (i) a relatively narrow, near tip 'fracture process zone' corresponding to a strong excursion of friction away from the steady-state $(\Delta f \gg b)$, followed by (ii) an approximately steady-state conditions $(\Delta f \approx 0)$ in the bulk of the slipping patch, i.e. away from the fracture tip. It is this type of the fracture-like behavior that led J.R. Rice [25] to advocate for applying the 'small scale yielding' (s.s.y.) concept of fracture mechanics [26] to model rate-state slip transients as singular cracks characterized by the simplified, steady-state frictional resistance in the bulk of the fracture, and by a stress singularity at the fracture tip with the intensity defined by the fracture energy $G_{c}$ (the rate of the energy dissipation within the near tip process zone when overcoming the frictional resistance $\Delta f$ in excess of the steady-state value). Recent study [27] landed further support to the idea by explicitly identifying the energy release rate into the tip region of numerically simulated ruptures on a rate-state fault.

Systematic application of the outlined fracture mechanics concepts to the analysis of the ratestate frictional ruptures have been, however, hampered by the lack of the robust prediction of the fracture energy and its dependence on the characteristics of the near tip region (e.g., rupture front speed $v_{r}$ ) over the entire spectrum of slip, from slow to fast. Notwithstanding is a suggestion $[9,10]$ to use the frictional state evolution in response to a step-increase in slip velocity (approximating the passage of the rupture front) as a proxy to calculate the fracture energy. Application of this ad hoc approximation remains challenging given that the 'slip velocity step' is a priory unknown, and, furthermore, not well-defined, as the slip rate behind the rupture front may exhibit significant spatial and temporal variability (e.g., Figure $1 \mathrm{c}, \mathrm{c}^{\prime}$ ).

In this work we consider the near-tip modeling of the rate-state frictional rupture ${ }^{1}$ and solve for the fracture energy $G_{c}$ and the peak stress perturbation carried by the rupture front as functions of the instantaneous rupture front speed $v_{r}$, which can assume any value, from slow / aseismic to fast /

\footnotetext{
${ }^{1}$ of the kind used previously in $[28,7]$ to determine fracture energy associated with co-seismic fault weakening by thermal pressurization and flash heating of frictional asperities
} 
seismic. We then use the s.s.y approach to develop an Equation of Motion (EoM) for slip transients driven on a 1D fault by a combination of two types of loading: (i) a uniform background overstress $\Delta f_{o}$ (the initial fault stress-ratio in excess of the steady-state friction value at the ambient fault sliding velocity), and (ii) localized fault strength perturbation at the slip 'hypocentral region (due to, e.g., a fluid injection) quantified by the time-varying magnitude of the net Coulomb hypocentral force $\Delta T(t)$. General properties of slip transients, as predicted by the EoM and validated by a number of fully numerical slip simulations (such as in Figure 1), are discussed when either the magnitude of the Coulomb force $\Delta T$ or its rate $\Delta T$ is a fixed constant. Particularly, we show that the maximum run-out distance of the aseismic slip transient on a rate-strengthening fault is $\propto \Delta T^{2}$ when $\Delta T=$ const, while the long-term run-out speed of the slip front is $\propto \Delta T$ when $\Delta T=$ const.

For fault slip driven by fluid injection, the assumption of the localized nature of strength perturbation stems from numerical modeling observations $[20,21]$ and inferences from in situ experiments $[29,30]$ that aseismic slip can significantly outpace pore pressure diffusion along the fault. The resulting Coulomb hypocentral force, $\Delta T \approx f_{0} \int \Delta p d z$ with $f_{0}=$ a representative value of friction there, is a simple proxy of (proportional to) the fluid volume injected into the fault zone - a result previously established by [23] for the particular case of a injection into a hydraulically sealed reservoir (such as Scenario 1 in Figure 1, when no fluid diffusion along the fault is allowed), but shown here to be generally valid for arbitrary injection history into a permeable fault. As such, EoM allows to track the maximum run-out distance of the aseismic slip in terms of the total injected fluid volume, and maximum slip front speed in terms of the average injection rate for an ongoing injection.

We finish the paper by drawing some conclusions and suggesting possible extensions of this work.

\section{Near Rupture Front Considerations}

\subsection{Rate-state friction}

The rate- and state-dependent friction can be written as [25]

$$
f=f_{\operatorname{dir}}(V)+\Theta, \quad f_{\operatorname{dir}}(V)=f_{o}+a \ln \frac{V}{V_{o}}
$$

where $f_{\text {dir }}(V)$ is the so-called 'direct-effect' term, which governs the instantaneous response of friction to a change in the slip rate $V=d \delta / d t$ from the ambient fault state characterized by slip rate $V_{o}$ and background stress ratio $f_{o}=\tau_{o} / \bar{\sigma}_{o}$, while $\Theta$ is the so-called 'state' term, which governs the dependence of friction on history of slip. Evolution of the state $\Theta$ with either incurred slip $\delta$ or time $t$ is driven by the departure of friction $f$ from its instantaneous, slip-rate-dependent steady state value $f_{s s}(V)$, denoted as

$$
\Delta f=f-f_{s s}(V)
$$

that can be generally written as

$$
\frac{d \Theta}{d \delta}=-\frac{1}{L} \Psi(\Delta f)
$$

Here $L$ is the state evolution distance (characteristic slip over which the change of the state takes hold) and function $\Psi(\Delta f)$ is such that $\Psi(0)=0$ and the sign of $\Psi(\Delta f)$ is that of $\Delta f$, or, in other 

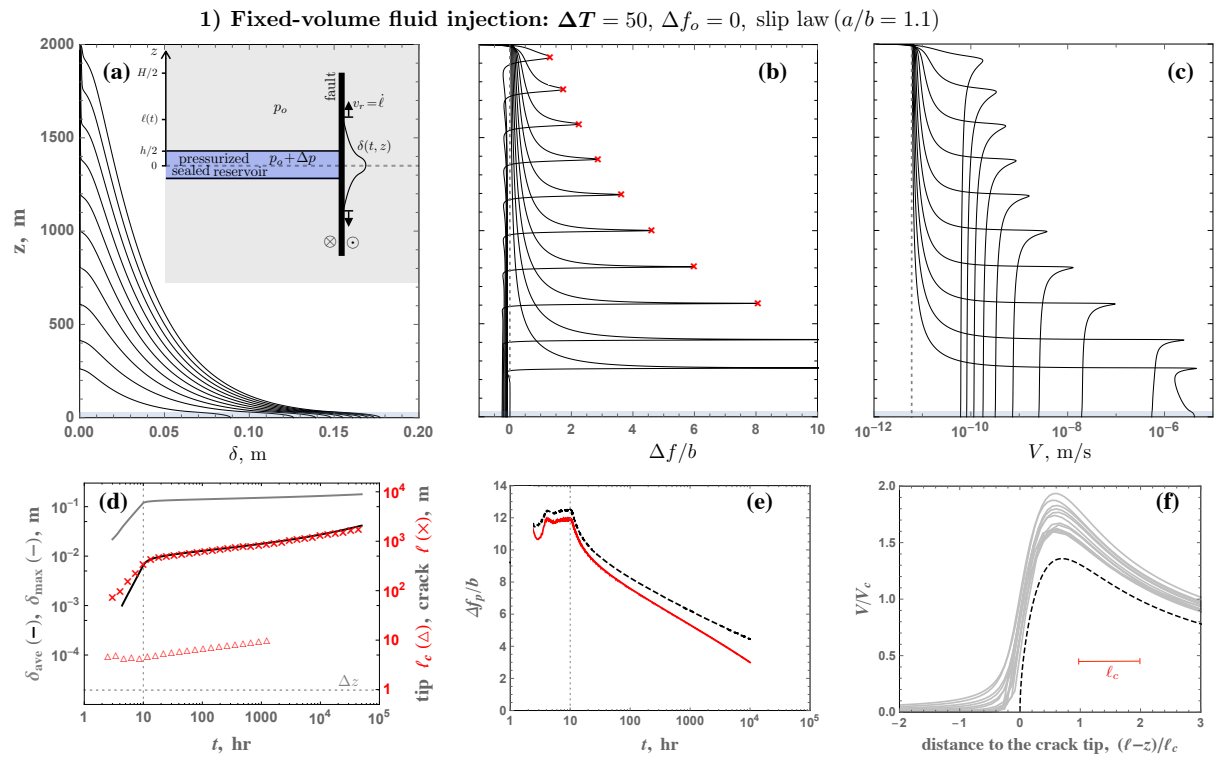

2) Fixed-volume-rate fluid injection: $\dot{\Delta} \boldsymbol{T}=10^{4}, \Delta f_{o}=0$, slip law $(a / b=1.1)$
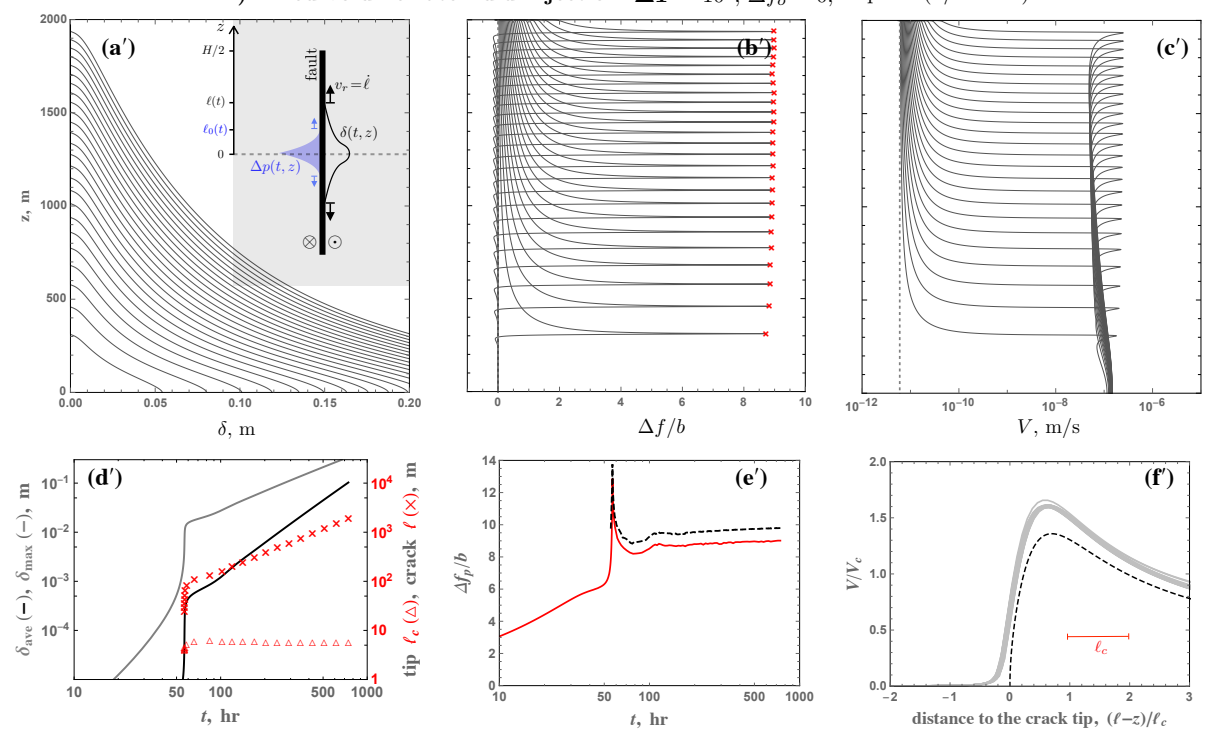

Figure 1: Symmetric slip development on a fault characterized by a rate-strengthening, rate-state friction in response to two scenarios: 1) a fixed-volume injection into a sealed-off reservoir abated by a fault (a-f) and $\mathbf{2})$ a fixed-volumetric-rate injection into a permeable fault (a'-f'). Snapshots of slip, (a), departure of the stress ratio (friction) from the stready-state, $\Delta f=f-f_{\mathrm{ss}}(V)$, (b), and slip rate, (c), are shown at $4 \mathrm{~mm}$ increments of the fault average slip $\delta_{\text {ave }}(t),(\mathbf{d})$. The front of the slipping patch ('crack'), $z= \pm \ell(t)$, is defined as the location of the peak friction change, $\Delta f_{p}$, shown by ' $\times$ ' in (b), while the evolution of the crack half-length $\ell(\times)$ and of the crack tip 'process zone' size $\ell_{c}(\triangle)$ with time $t$ shown in $(\mathbf{d})$ and $\Delta f_{p}(t)$ in (e). Approximate collapse of the slip rate profiles from (c) when rescaled in the fracture tip scaling $\left(V / V_{c}\right)$ and centered on the moving fracture tip (with the scaled distance to the tip $x=(\ell(t)-z) / \ell_{c}$ ), is shown in (f), where it is contrasted to the universal fracture tip solution (dashed line). (The scales $V_{c}$ and $\ell_{c}$ are given by universal functions of the instantaneous rupture front speed $v_{r}=d \ell / d t$ as defined in the text). The fracture tip solution prediction of $\Delta f_{p}$ is shown in (e) by dashed line.

Geometry: $H=4 \mathrm{~km}, h=60 \mathrm{~m}$ (scenario 1); discretization: $\Delta z=2^{-13} H$; elasticity: $\mu=30 \mathrm{GPa}$; friction: $a / b=1.1, b=0.01, L=1 \mathrm{~mm}, \ell_{b}=\left(\mu / b \bar{\sigma}_{o}\right) L=50 \mathrm{~m}$; initial fault state: $V_{o}=6 \times 10^{-12}$ $\mathrm{m} / \mathrm{s}, f_{o}=0.5+\Delta f_{o}$, zero overstress $\Delta f_{o}=0, \bar{\sigma}_{o}=\sigma_{o}-p_{o}=60 \mathrm{MPa}$; perturbation (1): constant excess resevoir pore pressure $\Delta p=50.7 \mathrm{MPa}$ (after 10-hour ramp-up period) corresponding to constant hypocentral Coulomb force $\Delta T=\int_{-\infty}^{\infty} f \Delta p d z \approx 50 \times \mu L$; pertrubation (2): constant fluid injection rate (per unit out-of-plane injection interval and unit permeable fault zone thickness) $q=3.7 \times 10^{-7} \mathrm{~m}^{3} / \mathrm{m}^{2} \mathrm{~s}$ corresponding to constant hypocentral Coulomb force-rate $\Delta T \approx 10^{4} \times \mu V_{o}$ (pore compressibility $\beta=1 / \mathrm{GPa}$ and hydraulic diffusivity $\alpha=0.01 \mathrm{~m}^{2} / \mathrm{s}$ of fault rocks). 
words, a positive / negative change $\Delta f$ pf friction from the steady state leads to a decreasing / increasing $\Theta$ with slip, and, thus, a tendency of the friction to evolve towards the steady state.

The state evolution function $\Psi(\Delta f)$ can be taken in either the 'slip law' or the 'ageing law' form $^{2}[4]$

$$
\Psi(\Delta f)=\Delta f \quad(\text { slip law }), \quad \Psi(\Delta f)=b\left[1-\exp \left(-\frac{\Delta f}{b}\right)\right] \quad(\text { aging law })
$$

The two laws are equivalent when that departure is small, $|\Delta f| / b \ll 1$. The logarithmic dependence of the steady-state friction $f_{s s}$ on the slip rate, $d f_{s s} / d V=(a-b) / V$ has been suggested by low velocity laboratory frictional sliding experiments $[3,31,5]$, and manifest either as a small rateweakening (RW), when $a-b<0$, or small rate-strengthening (RS), when $a-b>0$, of the steady-state fault friction with $|a-b| \ll 1$. It will be convenient to write the steady state friction expression in terms of the ambient fault state, $V_{o}$ and $f_{o}$,

$$
f_{s s}(V)=\left(f_{o}-\Delta f_{o}\right)+(a-b) \ln \frac{V}{V_{o}}
$$

where by definition (2), $f_{o}-\Delta f_{o}=f_{s s}\left(V_{o}\right)$ is the value of the steady state friction at $V_{o}$, and $\Delta f_{o}$ is the ambient value of the fault under $(<0)$ or over $(>0)$ stress ratio.

Rewriting (1) with help of (2) as $\Delta f=\Delta f_{\text {dir }}+\Theta$, where $\Delta f_{\text {dir }}=\Delta f_{o}+b \ln \left(V / V_{o}\right)$, and then differentiating with regards to slip, we get

$$
\frac{d \Delta f}{d \delta}=\frac{d \Delta f_{\operatorname{dir}}}{d \delta}-\frac{\Psi(\Delta f)}{L}
$$

\section{$2.2 \quad$ Near rupture tip solution}

Consider now evolution of slip near the 'front' of expanding slipping patch/rupture as exemplified in Figure 1. Although such front is not mathematically sharp, it can be easily identified as a narrow band of very large but finite slip rate gradient (Figure 1c) and a sharp peak in the friction departure $\Delta f$ form the steady-state (Figure 1b). Upon arrival of the front at a given point of the fault, the friction increases by the direct effect, i.e. $f \approx f_{\text {dir }}(V)$, to the level significantly above the instantaneous steady-state value $f_{s s}(V)$, until the fault has slipped a distance comparable to $L$ and the emerging state-evolution reverses the friction towards the new steady-state value (Figure 1b). The corresponding peak change of friction $\Delta f_{p}$ from the steady-state is then constrained by the direct-effect friction approximation on one hand, and by requiring $d \Delta f / d \delta=0$ in (6) on the other,

$$
\Delta f_{p} \approx \Delta f_{\mathrm{dir}}\left(V_{f_{p}}\right), \quad\left(\frac{d \Delta f_{\mathrm{dir}}}{d \delta}=b \frac{d \ln V}{d \delta}\right)_{f_{p}}=\frac{\Psi\left(\Delta f_{p}\right)}{L}
$$

where $V_{f_{p}}$ is the slip rate value at the peak departure from the steady-state (different from the peak value of the slip rate attained further behind the rupture front). In order to solve (7) for the $\Delta f_{p}$ and $V_{f_{p}}$, we need to know the slip evolution, or more specifically that of $d \ln V / d \delta$.

The subsequent post-peak evolution of friction towards the steady-state attained away from the rupture front, but at distances still small compared to the rupture length, can be modeled by

\footnotetext{
${ }^{2}$ Note that the ageing-law form of $\Psi$ given here relies on the classical, low-velocity-type of the steady state friction dependence on $V$, while the slip-law form of $\Psi$ is applicable for any type of the steady-state friction dependence on $V$ (e.g. high-velocity, flash heating type, etc)
} 
a semi-infinite crack with the mathematically sharp tip steadily advancing at the instantaneous value of the rupture velocity $v_{r}$. The solution is governed by the elastic equation of stress transfer for a semi-infinite crack written in the coordinate $x=v_{r} t-z$ moving with the crack tip (see Sup. Material for details)

$$
\Delta f(x)=\frac{\bar{\mu}\left(v_{r}\right)}{2 \pi \bar{\sigma}} \int_{0}^{\infty} \frac{d \delta / d x^{\prime}}{x^{\prime}-x} d x^{\prime}, \quad V(x)=v_{r} \frac{d \delta}{d x}
$$

where $\bar{\mu}=\mu g\left(v_{r}\right)$ is the apparent shear modulus which decreases with $v_{r}$ from the quasi-static value given by $\mu$ for the mode III and $\mu /(1-\nu)$ for the mode II fracture to zero when $v_{r}$ is approaching a limiting velocity given by the shear wave speed $c_{s}$ in mode III and the Raleigh speed $c_{R}$ in mode II [32]. E.g., $g=\sqrt{1-\left(v_{r} / c_{s}\right)^{2}}$ in mode III. Evolution of the frictional change $\Delta f$ from steady-state follows from the post-stress peak form of $(6)$, where the state evolution $-\Psi(\Delta f) / L$ dominates over the direct-effect $d \Delta f_{d i r} / d \delta$, and, thus, $d \Delta f / d \delta \approx-\Psi(\Delta f) / L$. Integrating the latter, one obtained the following post-peak slip-weakening relations

$$
\frac{\Delta f}{\Delta f_{p}} \approx \exp \left(-\frac{\delta}{L}\right) \quad(\operatorname{slip} \text { law }), \quad \frac{\Delta f}{\Delta f_{p}} \approx \frac{b}{\Delta f_{p}} \ln \left[1+\left(\exp \frac{\Delta f_{p}}{b}-1\right) \exp \left(-\frac{\delta}{L}\right)\right] \quad(\text { aging law })
$$

The ageing-law relation is parametrized by $\Delta f_{p} / b$, and can be shown to asymptotically coincide with the exponential slip-weakening form of the slip-law relation when $\Delta f_{p} / b \ll 1$, while it becomes a linear slip-weakening $\Delta f=\Delta f_{p}-b \delta / L$ when $\Delta f_{p} / b \gg 1$ (Figure 2a). Furthermore, both ageing- and slip- law weakening relations are of exponential slip-weakening form for large enough slip $\delta \gg L$, but with different prefactors ${ }^{3}$, which then leads to a well-defined fracture energy $G_{c}=\int_{0}^{\infty} \Delta f \bar{\sigma} d \delta$, as follows,

$$
G_{c}=\Delta f_{p} \bar{\sigma} L \quad(\text { slip law }), \quad G_{c}=-\operatorname{Li}_{2}\left(1-\exp \frac{\Delta f_{p}}{b}\right) \times b \bar{\sigma} L \quad(\text { aging law })
$$

and $\mathrm{Li}_{2}$ is the polylog function [33]. It is instructive that for small $\Delta f_{p} / b$, the fracture energy of the ageing-law friction is asymptotically the same as that for the slip law and, therefore, linear in $\Delta f_{p}$, while for large $\Delta f_{p} / b$ the ageing-law fracture energy increases much more rapidly (quadratically) with $\Delta f_{p}, G_{c} / b \bar{\sigma} L=0.5\left(\Delta f_{p} / b\right)^{2}$ (Figure $2 \mathrm{~b}$ ).

The solution of the sharp-rupture tip problem (8-9) for the normalized friction change $\Delta f / \Delta f_{p}$, slip rate $V / V_{c}$, and $\operatorname{slip} \delta / \delta_{c}$ with the normalized distance from the tip $x / \ell_{c}$ is obtained using a method based on Gauss-Chebyshev numerical calculus [34] and shown on Figure 3 for the slip and ageing laws of the rate-state friction ${ }^{4}$, respectively. Here the characteristic slip weakening distance $\delta_{c}$, slip rate $V_{c}$, and lengthscale $\ell_{c}$ are defined by

$$
\delta_{c}=\frac{G_{c}}{\Delta f_{p} \bar{\sigma}}, \quad V_{c}=\frac{\Delta f_{p} \bar{\sigma}}{\bar{\mu}} v_{r}, \quad \ell_{c}=\frac{\bar{\mu}}{\Delta f_{p} \bar{\sigma}} \delta_{c} .
$$

The normalized solution is parameter-less for the slip law, for which $G_{c}=\Delta f_{p} \bar{\sigma} L$ and $\delta_{c}=L$. In the case of the ageing law, the normalized solution is parameterized by $\Delta f_{p} / b$, since $\delta_{c} / L=$ $G_{c} /\left(\Delta f_{p} \bar{\sigma} L\right)$ is a function of $\Delta f_{p} / b$ following from (10.2), varying between 1 (when $\Delta f_{p} / b \ll 1$ ) and $0.5 \Delta f_{p} / b$ (when $\Delta f_{p} / b \gg 1$ ). The slip rate (Figure $3 \mathrm{a}$ ) is seen to evolve with distance from the

\footnotetext{
${ }^{3}$ For the ageing law, $\Delta f \approx b\left(\exp \left(\Delta f_{p} / b\right)-1\right) \exp (-\delta / L)$ when $\delta / L \gg 1$.

${ }^{4}$ The slip law solution is mathematically equivalent to the near-tip, underained-adiababtic solution for the rupture driven by the thermal pressurization obtained in [28].
} 

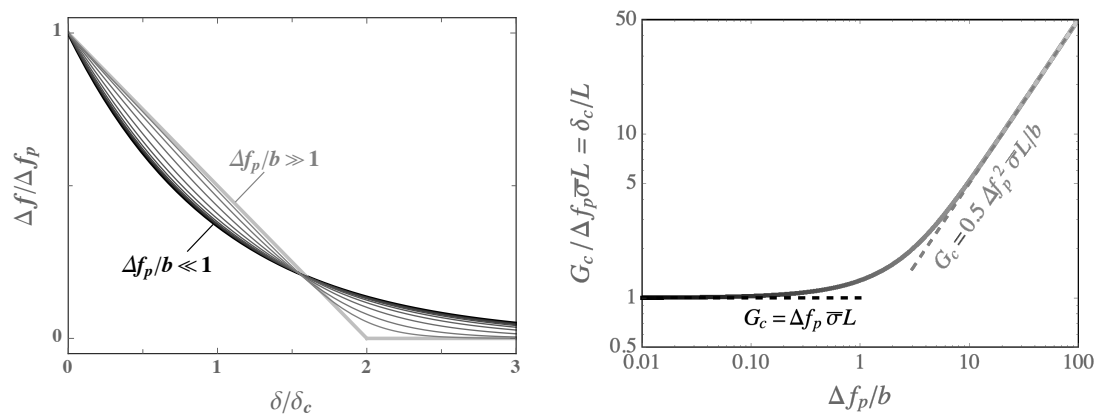

Figure 2: The post-peak slip-weakening near the rupture front, $\Delta f / \Delta f_{p}$ vs. $\delta / \delta_{c}\left(\Delta f_{p}\right)$, for various $\Delta f_{p} / b$ values, (a), and normalized fracture energy $G_{c} /\left(\Delta f_{p} \bar{\sigma} L\right)$ (and slip weakening distance $\delta_{c} / L$ ) as a function of $\Delta f_{p} / b,(\mathbf{b})$, for the ageing law of rate-state friction. The post-peak evolution in the case of the slip law of rate-state friction for arbitrary $\Delta f_{p} / b$ is identical to the limit $\Delta f_{p} / b \ll 1$ of the ageing case, see solid and dashed black lines in (a) and (b), respectively.

rupture front from the near, $V / V_{c}=k_{0}\left(x / \ell_{c}\right)^{1 / 2}$ when $x \ll \ell_{c}$, to the far, $V / V_{c}=\left(2 / \pi^{1 / 2}\right)\left(\ell_{c} / x\right)^{1 / 2}$ when $x \gg \ell_{c}$, field asymptotes, while attaining the peak value $\sim V_{c}$ at an intermediate distance $x \sim \ell_{c}$. The the near-field prefactor $k_{0}$ in the ageing law solution is moderately varying with $\Delta f_{p} / b$ between 1.845 and 1.614 (inset in Figure $3 \mathrm{a}$ ), while it is a constant $\left(k_{0} \approx 1.845\right)$ in the slip-law case. The far-field corresponds to the classic LEFM singularity for a fracture characterized by a finite fracture energy $G_{c}$.

\subsection{Peak friction change and fracture energy}

The post-peak 'sharp-tip' solution allows to determine the fracture energy of the frictional process near the rupture front, (10), in terms of the peak value $\Delta f_{p}$ of friction departure from the steady state. The latter depends on the pre-peak evolution of slip, which is neglected in the sharp-tip solution. To determine $\Delta f_{p}$, we will assume that the relationship between the slip and slip rate in the near field of the sharp-tip solution ${ }^{5}, \delta / \delta_{c}=\left(2 / 3 k_{0}^{2}\right)\left(V / V_{c}\right)^{3}$, can be approximately extrapolated to the pre-peak region. If so, conditions (7) at the peak friction change can be rewritten as

$$
\Delta f_{p}=\Delta f_{o}+b \ln \frac{V_{f_{p}}}{V_{o}}, \quad \frac{b}{\delta_{c}} \frac{k_{0}^{2}}{2}\left(\frac{V_{c}}{V_{f_{p}}}\right)^{3}=\frac{\Psi\left(\Delta f_{p}\right)}{L}
$$

Accounting in the above for the dependence (11) of scales $V_{c}$ and $\delta_{c}$ on $v_{r}$ and $G_{c}\left(\Delta f_{p}\right)$, we can solve for the rupture velocity $v_{r}$ as a function of the peak friction change $\Delta f_{p}$ :

$$
\frac{v_{r} / \bar{v}_{o}}{g\left(v_{r} / c_{s}\right)}=\mathscr{V}\left(\Delta f_{p} / b\right), \quad \text { where } \quad \bar{v}_{o}=\exp \left(-\Delta f_{o} / b\right) \frac{\mu}{b \bar{\sigma}_{o}} V_{o}
$$

is a characteristic rupture velocity scale embodying the dependence on the fault ambient conditions, and function $\mathscr{V}\left(\Delta f_{p} / b\right)$ is given by

$$
\mathscr{V}(\zeta)=\kappa_{0} \frac{e^{\zeta}}{\zeta^{2 / 3}} \quad(\text { slip law }), \quad \mathscr{V}(\zeta)=\kappa_{0}(\zeta) \frac{-e^{\zeta}\left(1-e^{-\zeta}\right)^{1 / 3} \mathrm{Li}_{2}^{1 / 3}\left(1-e^{\zeta}\right)}{\zeta^{4 / 3}} \quad \text { (aging law) }
$$

\footnotetext{
${ }^{5}$ as follows from integrating the slip-rate near-field asymptote $V \propto x^{1 / 2}$ for slip $\delta \propto x^{3 / 2}$
} 

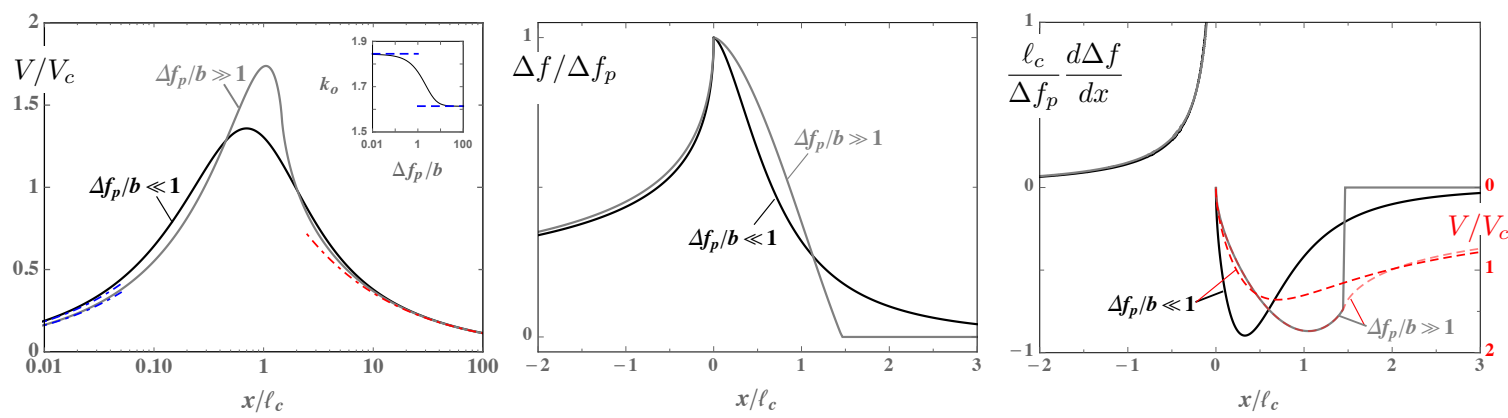

Figure 3: The post-stress-peak, sharp rupture tip solution for the normalized (a) slip rate $V$, (b) stress ratio change $\Delta f$, and (c) stress gradient $d \Delta f / d x$ with distance from the tip $x$ for the ageing law of the rate-state friction in the limit of small $\left(\Delta f_{p} / b \ll 1\right)$ and large $\left(\Delta f_{p} / b \gg 1\right)$ peak friction departure from the steady-state. Dotdashed lines in (a) show the near and the far fields of the slip rate, with the corresponding near field prefactor $k_{0}$ shown in the inset as a function of $\Delta f_{p} / b$. Dashed lines in (c) show the normalized slip rate from (a) to compare to the normalized stress gradient profiles. The rupture tip solution for the slip law of the rate-state friction for arbitrary value of $\Delta f_{p}$ is given by the ageing law solution with $\Delta f_{p} / b \ll 1$. The length $\ell_{c}$ and slip rate $V_{c}$ scales are defined in terms of the fracture energy (or, conversally, in terms of $\Delta f_{p}$ ) in (11).

Here $\kappa_{0}=\left(2 / k_{0}^{2}\right)^{1 / 3}$ is a constant $\approx 0.838$ for the slip law and is a function with values between $\kappa_{0}(0) \approx 0.838$ and $\kappa_{0}(\infty) \approx 0.916$ for the ageing law. The corresponding value of friction at the peak change follows from $f_{p}=\Delta f_{p}+f_{s s}\left(V_{f_{p}}\right)=(a / b)\left(\Delta f_{p}-\Delta f_{o}\right)$.

Equation (13) provides an implicit form of the dependence of the peak friction change $\Delta f_{p}$, and, therefore, of the fracture energy $G_{c},(10)$, on the rupture speed $v_{r}$. This is plotted in Figure 4 , showing $\Delta f_{p} / b$ and $G_{c} / b \sigma L$ vs. the scaled rupture velocity function ${ }^{6} v_{r} / \bar{v}_{o} g\left(v_{r} / c_{s}\right)$. We note that the given scaled relations are parameter-free (i.e. universal), and can be used to unravel the full dependence of the solution for the fracture energy on the problem parameters. The solution has distinct decreasing $\left(\Delta f_{p} / b \lesssim 1\right)$ and increasing $\left(\Delta f_{p} / b \gtrsim 1\right)$ branches, which characteristically correspond to distinct slip rate $V_{f_{p}}$ values that are comparable to and larger than the fault ambient slip rate $V_{o}$, respectively. We anticipate that the underlining sharp-crack-tip model does not hold for the former decreasing branch, and henceforth consider the increasing $\Delta f_{p}$ branch only. The solution is characterized by the minimum value of the fracture energy $G_{c, \min } / b \bar{\sigma} L=\Delta f_{p, \min } / b \approx$ 2.552 attained at $v_{r, \min } \approx 5.76 \bar{v}_{o}$, and by the approximately-logarithmic increase of $\Delta f_{p}$ for large values of $v_{r} / \bar{v}_{o}$. Interestingly, the $\Delta f_{p}$ solutions for the slip and ageing laws are at most few percent different, making them practically indistinguishable in log-scale of Figure 4. However, the fracture energy solutions for the two friction laws are drastically different when $v_{r} \gg \bar{v}_{o}$ (and $\left.\Delta f_{p} \gg b\right)$, with the ageing-law $G_{c}$-values much larger than the slip-law ones, owing to the different dependence (10) of the fracture energy on the peak friction change $\Delta f_{p}$ for the two laws.

Equation (13) is implicit in $\zeta=\Delta f_{p} / b$, and convenient explicit approximations to its solution are obtained by applying an iterative process $\zeta^{(i+1)}=\ln \left(\mathscr{V} / \chi\left(\zeta^{(i)}\right)\right.$ to invert $\mathscr{V}(\zeta)=\chi(\zeta) e^{\zeta}$ (where, e.g., $\chi(\zeta)=\kappa_{0} / \zeta^{2 / 3}$ for the slip law). Selecting the (seed) zeroth order approximation as $\zeta^{(0)}(\mathscr{V})=$ $\ln \left(\mathscr{V} / \kappa_{0}\right)$ yields an excellent approximation by the 2 nd iteration, $\zeta^{(2)}(\mathscr{V})$ (Figure 4). Although $\zeta^{(0)}(\mathscr{V})$ provides a rather crude approximation, it does allow for a fair bit of interpretational

\footnotetext{
${ }^{6}$ For aseismic ruptures, $g \approx 1$ and the latter simply becomes a normalized rupture velocity $v_{r} / \bar{v}_{o}$
} 


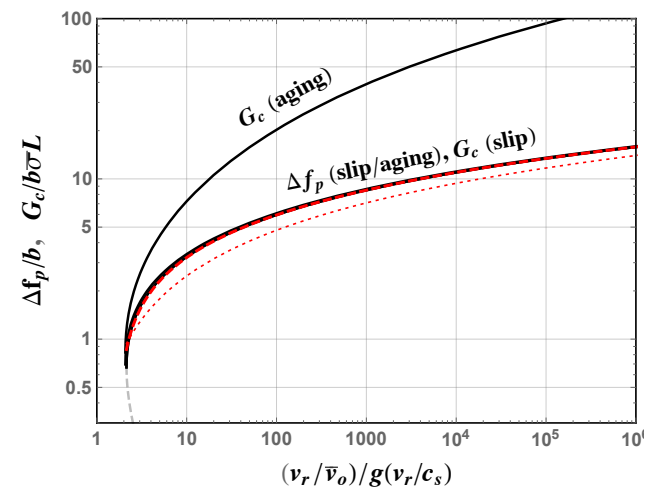

Figure 4: Normalized peak friction departure from the steady state $\Delta f_{p}=\left(f-f_{s s}(V)\right)_{\text {peak }}$ and fracture energy $G_{c}$ as functions of the normalized rupture speed $v_{r}, v_{r} /\left(\bar{v}_{o} g\left(v_{r} / c_{s}\right)\right)$, where $\bar{v}_{o}=\exp \left(-\Delta f_{o} / b\right)(\mu / b \bar{\sigma}) V_{o}$ embodies the dependence on the fault ambient conditions and $g\left(v_{r} / c_{s}\right)$ is the elastodynamic factor $(g(0)=1$ and $g(1)=0$ in mode III). The increasing / decreasing solution branch is shown by solid / opaque line. Red dotted and dashed lines show the simplified 0th (Eq. (14)) and 2nd iteration approximations of the increasing $\Delta f_{p}$ solution.

simplicity. Indeed, in this approximation (13) reduces to

$$
\Delta f_{p} \approx \Delta f_{o}+b \ln \frac{v_{r} / v_{o}}{\kappa_{0} g\left(v_{r} / c_{s}\right)} \quad\left(\Delta f_{p} \gg b\right)
$$

with the rupture velocity scale $v_{o}=(\mu / b \bar{\sigma}) V_{o}$ is now determined by the fault ambient slip rate $V_{o}$ only, and for the absolute value of the 'at peak' friction

$$
f_{p} \approx f_{o}+a \ln \frac{v_{r} / v_{o}}{\kappa_{0} g\left(v_{r} / c_{s}\right)} \quad\left(\Delta f_{p} \gg b\right)
$$

Comparing the above approximations with the first in (12), the quantity under the logarithm is the corresponding approximation of the slip rate at the peak friction change scaled by the ambient value, i.e. $V_{f_{p}} / V_{o}$.

The developed universal tip solution (Figure 3) in the process zone scaling (11) and the corresponding solution for the peak friction change (Figure 4) are validated by comparing to the near tip region of full numerical solutions for the slow-slip transients on a fault with slip-law, rate-state friction, Figure 1(f, $\left.\mathrm{f}^{\prime}\right)$ and $1\left(\mathrm{e}, \mathrm{e}^{\prime}\right)$. (Similar comparisons for other slow-slip numerical examples for the slip-law and ageing-law of fault friction are shown in Sup. Figures 1-3). Particularly, the numerical slip rate profiles with peak velocities varying over several orders of magnitude over the event propagation time (Figure 1c, $\mathrm{c}^{\prime}$ ) are seen to collapse when using the tip scaling (11) and approximately match the universal tip solution profile (Figure 1f, $\mathrm{f}^{\prime}$ ). The latter somewhat underestimates the former, but the agreement between the two improves with increasing rupture speed, or, conversely, deteriorates as the slip front and slip rate decelerate in the case of constant hypocentral force (which also contributes to a moderate scatter of the rescaled numerical profiles, see also Sup. Figure 2 for examples of more 'vigorous' slow slip transients on an overstressed fault). Similarly, the peak friction change $\Delta f_{p}$, and hence the fracture energy, extracted from the numerical solutions compare well to, if somewhat overestimated by, the rupture-speed-dependent 
prediction of the universal tip solution (Figure 1e, $\mathrm{e}^{\prime}$ ). The approximate nature of the universal tip solution is likely a consequence of the assumptions taken (i.e., neglected direct effect post-frictionpeak and the 'sharp' crack tip approximation, see further discussion in Sup. Materials), which could be relaxed in future work.

\section{Equation of Motion}

Examination of slip transients propagating on rate-state faults shows that the friction excursion away from the steady-state is mostly confined to a relatively narrow region near the leading edge of the rupture, while the friction is approximately at the steady-state within the rupture bulk. This suggests [25] that the rupture can be approximately modeled by that overcoming the steady-state frictional fault strength in the bulk of the slipping region and the finite fracture energy at the advancing front, with the latter is now an established function of the rupture velocity obtained from the universal near-tip solution.

\subsection{Fracture Mechanics of Slipping Patch Propagation}

The propagation of the slipping patch within the above assumptions is governed by a variant of the small scale yielding fracture mechanics [26, 32], stating that the energy release rate $G=\bar{K}^{2} /(2 \bar{\mu})$ at the propagating crack tip matches the the fracture energy $G_{c}=\Delta f_{p}\left(v_{r}\right) \bar{\sigma}_{o} L$ obtained in the preceding section. The dynamic stress intensity factor $\bar{K}$ can be written as a product $\bar{K}=k\left(v_{r}\right) K$ of the static stress intensity factor $K$ and a prefactor function $k\left(v_{r}\right)$ of the rupture velocity. The latter is only weakly dependent on the fracture configuration and the propagation history, and is approximated here by the form obtained from the self-similar, constant stress-drop symmetric crack expansion solution, which in the mode III case is given by $k\left(v_{r}\right)=\sqrt{1-v_{r}^{2} / c_{s}^{2}} / E\left(1-v_{r}^{2} / c_{s}^{2}\right)$ where $E$ is the complete elliptic integral of the 2 nd kind (e.g., [35] $)^{7}$. Thus, we can write the propagation condition in terms of the static stress intensity factor as

$$
K \approx K_{c}=\sqrt{2 \mu G_{c} g / k^{2}}
$$

where the fracture toughness $K_{c}$ is a uniquely defined function of the rupture velocity $v_{r}=\dot{\ell}$.

We consider homogeneous ambient fault conditions, characterized by the background shear $\tau_{b g, o}=f_{o} \bar{\sigma}_{o}$ and effective normal $\bar{\sigma}=\bar{\sigma}_{o}$ stresses, over which the rupture (slipping patch) propagation takes place either quasi-statically (aseismically) or dynamically from a hypocenter at $x=0$. In the hypocentral region the fault loading and/or strength may be perturbed from the ambient values, which is presumed to give either initial or continuous stimulation of fault slip. E.g., it can be a temporal hypocentral loading leading to nucleation of run away dynamic rupture on frictionally unstable fault (in which case it can be reasonably neglected for large time analysis of the rupture run-out), or it can be temporal or continual loading leading to aseismic slip propagation, as in, e.g., localized reduction of fault strength by pore pressure increase due to fluid injection. In either case, we going to assume that the spatial extent of the perturbed hypocentral region is small compared to the slipping patch size, which lands itself to be modeled as a net Coulomb force $\Delta T(t)$ acting at the hypocenter.

\footnotetext{
${ }^{7}$ To further substantiate the claim of the weak dependence of the $k\left(v_{r}\right)$ on a particular crack configuration, we observe that the expression $k\left(v_{r}\right)=\sqrt{1-v_{r} / c_{s}}$ for a semi-infinite non-uniformly propagating mode III crack (e.g., [32]) is quantitatively only marginally different from the one adopted here.
} 
Consequently, we adopt the following approximate decomposition for the static stress intensity factor

$$
K=K_{\Delta \tau}+K_{\Delta T}
$$

$K_{\Delta \tau}$ is the contribution to $K$ due to the generally spatially variable stress drop $\Delta \tau=\tau_{b g}-f \bar{\sigma}$ along the bulk of the patch, away from the possibly perturbed hypocentral region, such that $\tau_{b g}=\tau_{b g, o}=f_{o} \bar{\sigma}_{o}$ and $\bar{\sigma}=\bar{\sigma}_{o}$, and away from the rupture front, such that $f \approx f_{s s}(V)$,

$$
K_{\Delta \tau}=\sqrt{\frac{\ell}{\pi}} \int_{-\ell}^{+\ell} \frac{\left(f_{o}-f_{s s}(V)\right) \bar{\sigma}_{o}}{\sqrt{\ell^{2}-x^{2}}} d x=\Delta \tau_{\mathrm{eff}} \sqrt{\pi \ell},
$$

and the effective (equivalent uniform) stress drop $\Delta \tau_{\text {eff }}$ can be written in terms of the patch effective slip rate $V_{\text {eff }}$ as

$$
\Delta \tau_{\mathrm{eff}}=\left(f_{o}-f_{s s}\left(V_{\mathrm{eff}}\right)\right) \bar{\sigma}_{o} \quad \text { with } \quad f_{s s}\left(V_{\mathrm{eff}}\right) \equiv \frac{1}{\pi} \int_{-\ell}^{\ell} \frac{f_{s s}(V)}{\sqrt{\ell^{2}-x^{2}}} d x
$$

$K_{\Delta T}$ is the contribution to $K$ due to the hypocentral perturbation,

$$
K_{\Delta T} \approx \frac{\Delta T}{\sqrt{\pi \ell}}
$$

where the net hypocentral Coulomb force

$$
\Delta T=\int_{-\ell_{0}}^{+\ell_{0}}\left(\widetilde{\tau}_{b g}-f_{s s}(V) \widetilde{\sigma}\right) d x \approx \Delta T_{b g}-f_{0} \Delta N \quad \text { with } \quad f_{0}=f_{s s}\left(V_{\text {eff }, 0}\right)=\frac{1}{2 \ell_{0}} \int_{-\ell_{0}}^{+\ell_{0}} f_{s s}(V) d x
$$

embodies the effects of perturbations $\widetilde{\tau}_{b g}=\tau_{b g}-f_{o} \bar{\sigma}_{o}$ and $\widetilde{\sigma}=\bar{\sigma}-\bar{\sigma}_{o}(=-\Delta p)$ of the background shear and effective normal stresses, respectively, assumed to be confined to a small hypocentral region $|x|<\ell_{0}$ with $\ell_{0} \ll \ell$. An approximate expression for $\Delta T$ is given in (21) in terms of the assumed-to-be-known net background shear $\Delta T_{b g}=\int_{-\ell_{0}}^{+\ell_{0}} \tilde{\tau}_{b g} d x$ and net effective normal $\Delta N=$ $\int_{-\ell_{0}}^{+\ell_{0}} \tilde{\sigma} d x$ perturbative forces, and, in general, unknown value of the hypocentral friction $f_{0}$.

Using (17) with (18) and (20), the propagation condition (16) can be written as

$$
\Delta \tau_{\text {eff }}+\frac{\Delta T}{\pi \ell}=\frac{K_{c}}{\sqrt{\pi \ell}} \quad \text { with } \quad \Delta \tau_{\text {eff }}=\left(f_{o}-f_{s s}\left(V_{\text {eff }}\right)\right) \bar{\sigma}_{o}
$$

We next derive the bulk effective slip rate $V_{\text {eff }}$, defined in (19), as a function of $\ell$ and $\dot{\ell}$ by considering the slip rate distribution along the slipping patch, while similar derivation of the hypocentral slip rate $V_{\text {eff }, 0}$ given in Sup. Materials ${ }^{8}$, and, thus, establish (22) as the rupture front equation-ofmotion (EoM).

\subsection{Slip Rate Distribution and $V_{\text {eff }}$}

In line with the crack loading decomposition, the slip along the patch is approximated by the superposition of that due to the effective uniform stress drop $\Delta \tau_{\text {eff }}, \delta_{\Delta \tau}$, and of that due to the hypocentral Coulomb force $\Delta T, \delta_{\Delta T}$,

$$
\delta \approx \delta_{\Delta \tau}+\delta_{\Delta T} \quad \text { with } \quad \delta_{\Delta \tau}=\frac{2 \Delta \tau_{\text {eff }}}{\hat{\mu}} \sqrt{\ell^{2}-x^{2}}, \quad \delta_{\Delta T}=\frac{2 \Delta T}{\pi \hat{\mu}} \cosh ^{-1} \frac{\ell}{|x|}
$$

\footnotetext{
${ }^{8}$ Note that the dependence of $\Delta \tau_{\text {eff }}$ on $V_{\text {eff }}$ is more essential to the accurate evaluation of EoM (22) than that of $\Delta T$ on $V_{\text {eff, }, 0}$, since the small changes of the former with effective slip rate are amplified in (22) by increasing function of crack length, either $\ell$ or $\sqrt{\ell}$, when comparing the EoM terms.
} 
Here $\hat{\mu}\left(v_{r}\right)=\mu g\left(v_{r}\right) / k\left(v_{r}\right)$ is an apparent shear modulus which monotonically decreases with $v_{r}$ from the quasi-static value given by $\mu$ in mode III and $\mu /(1-\nu)$ in mode II to a finite fraction thereof when $v_{r}$ is approaching a limiting velocity given by the shear wave speed $c_{s}$ in mode III and the Raleigh speed $c_{R}$ in mode II $[35,32]^{9}$. Specifically, for the mode III $g / k=E\left(1-v_{r}^{2} / c_{s}^{2}\right)$, in which $E$ is the complete elliptic integral of the 2nd kind, varies modestly between $(g / k)(0)=1$ to $(g / k)(1)=2 / \pi$. The corresponding slip rate distribution follows by time differentiation ${ }^{10}$ of $(23)$ and the use of $(22)$

$$
V \approx \frac{2 K_{c}}{\hat{\mu} \sqrt{\pi \ell}} \frac{\dot{\ell}}{\sqrt{1-x^{2} / \ell^{2}}}+\frac{2 \Delta T}{\pi \hat{\mu}} \cosh ^{-1} \frac{\ell}{|x|}
$$

Consider first evaluating the effective slip rate $V_{\text {eff }},(19)$, when either the rate of change of the hypocentral Coulomb force or of the fracture toughness is negligible. For the low-velocity (logarithmically dependent on $V$ ) friction, we can get

$$
V_{\mathrm{eff}}^{(\Delta T=0)}=4 \frac{K_{c} \dot{\ell}}{\hat{\mu} \sqrt{\pi \ell}}, \quad V_{\mathrm{eff}}^{\left(K_{c}=0\right)}=0.4569 \times \frac{\dot{\Delta T}}{\hat{\mu}}
$$

In the general case, we can obtain from (19), after some manipulations,

$$
V_{\text {eff }}=\mathscr{F}(\mathscr{P}) \frac{4 K_{c} \dot{\ell}}{\hat{\mu} \sqrt{\pi \ell}}, \quad \text { where } \quad \mathscr{P}=\frac{\dot{\Delta T} \sqrt{\pi \ell}}{K_{c} \dot{\ell}}
$$

is the non-dimensional parameter with the meaning of the normalized point-force-rate, and function $\mathscr{F}(\mathscr{P})$, defined by

$$
\ln \mathscr{F}(\mathscr{P})=\frac{1}{\pi} \int_{-1}^{+1} \ln \left(1+\frac{\mathscr{P}}{\pi} \sqrt{1-\xi^{2}} \cosh ^{-1} \frac{1}{|\xi|}\right) \frac{d \xi}{\sqrt{1-\xi^{2}}},
$$

and plotted in Sup. Figure 4, provides the transition with $\mathscr{P}$ from the toughness-dominated $(\mathscr{P} \ll 1$ or negligible $\Delta T), \mathscr{F} \sim \exp (\mathscr{P} / \pi) \approx 1$, to the point-force-rate-dominated $(\mathscr{P} \gg 1$ or negligible $K_{c}$ ), $\mathscr{F} \sim 0.1142 \times \mathscr{P}$, regime. General expression (26) for the bulk slip rate naturally reduces to the asymptotic ones given in $(25)$ when $\mathscr{P}$ is much smaller/larger than 1.

As a final note on the formulation of EoM, we acknowledge that the ambient slip rate on faults that are initially over or understressed, i.e. when $\Delta f_{o} \neq 0$, will evolve over a long (ambient) timescale $t_{o}=L / V_{o}$ towards the new steady-state value, thus leading to reduction of the absolute value of $\Delta f_{o}$ over the same timescale. This baseline evolution of $V_{o}$ and $\Delta f_{o}$ can in principle be inserted into the EoM considerations without any change to the latter, but are practically unnecessary when considering transient evolution of a 'stimulated' slip events on timescales shorter than $t_{o}$ (e.g., equal to $t_{o} \approx 5.3$ years for the fault parametrization in the numerical examples of slip transients considered in this work, such as Figure 1).

\footnotetext{
${ }^{9}$ This form of the apparent shear modulus is strictly valid for a self-similarly expanding crack with constant uniform stress drop. Here we tentatively assume that the solution for the crack driven by by a point-force can be formulated in terms of the same apparent modulus.

${ }^{10}$ assuming a slowly varying toughness $K_{c}$ and apparent modulus $\hat{\mu}$, and, thus, neglecting their rates when carrying differentiation
} 


\subsection{EoM predictions}

Let us make use of the following natural elasto-frictional scaling

$$
\frac{t}{t_{o}} \rightarrow t, \frac{\ell}{\ell_{b}} \rightarrow \ell, \frac{v_{r}}{v_{o}} \rightarrow v_{r}, \frac{V}{V_{o}} \rightarrow V, \frac{\Delta T}{\mu L} \rightarrow \Delta T, \frac{\Delta T}{\mu V_{o}} \rightarrow \dot{\Delta T}, \frac{G_{c}}{b \bar{\sigma}_{o} L} \rightarrow G_{c}, \frac{K_{c}}{b \bar{\sigma}_{o} \sqrt{\ell_{b}}} \rightarrow K_{c}
$$

defined in terms of the characteristic length $\ell_{b}=\left(\mu / b \bar{\sigma}_{o}\right) L$ and time $t_{o}=\ell_{b} / v_{o}=L / V_{o}$ scales. EoM (22), normalized with help of (28), reads

$$
\underbrace{\Delta f_{o} / b-(a / b-1) \ln V_{\mathrm{eff}}}_{\Delta \tau_{\mathrm{eff}} /\left(b \bar{\sigma}_{o}\right)}+\frac{\Delta T}{\pi \ell}=\frac{K_{c}}{\sqrt{\pi \ell}} \quad \text { with } \quad V_{\mathrm{eff}}=4 \dot{\ell} \frac{K_{c}}{\sqrt{\pi \ell}} \mathscr{F}\left(\frac{\Delta T}{\dot{\ell}} \frac{\sqrt{\pi \ell}}{K_{c}}\right)
$$

where the scaled fracture energy $G_{c}=G_{c}\left(e^{\Delta f_{o} / b} \dot{\ell} / g\right)$ (Figure 4) and toughness $K_{c}=\sqrt{2 G_{c} g / k^{2}}$ are functions of the scaled rupture velocity $v_{r}=\dot{\ell}$ (while $g$ and $k$ are the previously defined elastodynamic functions of $v_{r} /\left(c_{s} / v_{o}\right)$ with $g=k=1$ for aseismic slip). EoM is an implicit 1st order ordinary differential equation governing evolution of the rupture scaled half-length $\ell=\ell(t)$, which is parametrized by the direct-to-state friction parameters ratio $a / b$, ambient overstress $\Delta f_{o} / b$, scaled wave speed $c_{s} / v_{o}$ (only of importance for seismic ruptures), and scaled hypocentral force $\Delta T$ and its time-rate $\Delta T$.

Consider the case of constant hypocentral Coulomb force $(\Delta T=$ const, $\Delta T=0$, and $\mathscr{F}(0)=1$ ) for which the EoM does not depend explicitly on time, and is entirely described by a trajectory ${ }^{11}$ in the phase-plane $(\ell, \dot{\ell})$. The EoM phase trajectories are shown in Figure 5 for the case of the slip-law friction ${ }^{12}$ and for systematically varied frictional rate-dependence parameter $a / b$ (from rate-weakening, $a / b<1$, to rate-strengthening, $a / b>1$ ), Coulomb force $\Delta T$, and ambient fault overstress $\Delta f_{o} / b$.

For frictionally-stable faults (RN and RS panes of the figure), the overstressed conditions $\left(\Delta f_{o} / b=+1\right)$ are seen to support long-term expansion and acceleration of slow slip, as the rupture speed and the bulk slip rate $V_{\text {eff }}$ (shown by the color gradient) increase with run-out distance to eventually seismic values ${ }^{13}$. This eventual seismic transition on RS faults predicted to occur over exceedingly large slip run-outs, which are likely not practical to observe in nature. In the case of a finite hypocentral perturbation $(\Delta T>0)$, early-time development of slip is dominated by a possibly seismic (for large enough $\Delta T$ ) transient owing to an abrupt application of the Coulomb perturbation [19] followed by the rupture deceleration at intermediate times/lengths irrespective of the fault initial conditions (overstress value). This hypocentral-perturbation-dominated behavior eventually gives way to the long-term slip accelerating dynamics on overstressed faults, as already described in the above, while the slip-rate fizzles to the ambient value on under- and neutrallystressed faults $\left(\Delta f_{o} / b \leq 0\right)$, essentially signaling termination of the slip transient run-out ${ }^{14}$. EoM examination of the slip development on frictionally-stable faults with ageing-law of friction (phase plots on Sup. Figure 5) shows qualitatively similar but much slower dynamics owing to a much larger fracture energy when compared to the slip-law friction case at the same value rupture speed

\footnotetext{
${ }^{11}$ which can be easily integrated to obtain rupture front progression with time $\ell=\ell(t)$

12 phase-plots for the ageing-law of friction are shown in Sup. Figure 5

13 also observed by [21] but in the context corresponding to a constant rate of Coulomb force (constant rate of fluid injection into the fault in that study).

${ }^{14}$ The slightly accelerating long-term rupture predicted by the EoM for the case of neutrally stressed fault $\Delta f_{o}=0$ with $\Delta T>0$ corresponds to the slip-rate in bulk of the rupture asymptotically approaching the ambient fault value, and, thus, an artifact of application of EoM outside of its underline assumptions.
} 
(Figure 4). A practical estimate of the maximum slip run-out distance $\ell_{\max }$ on neutrally- and under- stressed $\left(\Delta f_{o} \leq 0\right)$, frictionally-stable $(a / b \geq 1)$ faults is obtained from the EoM by setting normalized bulk slip rate $V_{\text {eff }} \sim 1$ and corresponding $G_{c} \sim 1$, which leads to a simple quadratic equation for $\ell_{\max }$. At zero fault overstress,

$$
\Delta f_{o}=0: \quad \ell_{\max }=\Delta T^{2} / 2 \pi
$$

For frictionally-unstable, $R W$ faults (left pane of the figure), a rather complicated variety of rupture behaviors is suggested by the EoM, including the non-unique rupture velocity (simultaneous existence of the accelerating and decelerating solution branches) for large enough rupture run-out distance. The minimum run-out distance at which the non-uniqueness emerges may indicate the slip instability (earthquake nucleation) studied in the numerous previous works including $[9,10,11]$. Exploration of this possible connection is outside of the scope of this work, we do note however, that (i) care would need to be exercised in applying the crack-like EoM to earthquake nucleation on faults with slip-law rate-state friction, which were shown to support the pulse-like rupture propagation mode [10]; and, more optimistically, (ii) the critical slip run-out distance predicted by the EoM on initially steady-state faults $\left(\Delta f_{o}=0\right)$ with ageing-law of friction is very well approximated by $\ell_{\infty}=\ell_{b} /\left(\pi(1-a / b)^{2}\right)$, in agreement with the earthquake nucleations studies $[9,11]$.

In case of a constant rate of the hypocentral Coulomb force $(\Delta T>0)$, a useful approximation of the EoM (29) is obtained by differentiating in time and reasonably neglecting the rates of $K_{c}$ and $\Delta \tau_{\text {eff }}$ (since both the toughness and the bulk stress drop are only weakly dependent upon the time-varying rupture velocity)

$$
\underbrace{\Delta f_{o} / b-(a / b-1) \ln V_{\mathrm{eff}}}_{\Delta \tau_{\mathrm{eff}} /\left(b \bar{\sigma}_{o}\right)}+\frac{\Delta T}{\pi \dot{\ell}} \approx \frac{1}{2} \frac{K_{c}}{\sqrt{\pi \ell}}
$$

When $\dot{\Delta T}$ is a constant, EoM (31) does not explicitly depend on time, and, thus, is fully-described by a trajectory in the phase-plane. Figure 6 (Sup. Figure 6) shows the EoM solution trajectories for the slip-law (ageing-law) friction, for a range of values of the rate-dependence parameter $a / b$, ambient overstress $\Delta f_{o}$, and the rate of the hypocentral Coulomb force $\Delta T$, respectively. Focusing on the response of rate-strengthening faults (c) to the linear increase of the hypocentral Coulomb force with time, we observe that the initially overstressed faults $\left(\Delta f_{o}>0\right)$ support continually accelerating slip transients at large run-out distances similarly to the case with a constant Coulomb force (Figure 5). This simply suggests that the fault overstress (when present), not the hypocentral perturbation, dominates the slip response at large time/run-out. However, the large-run-out rupture behavior on neutrally- or under- stressed faults $\left(\Delta f_{o} \leq 0\right)$ is markedly different from that for a constant Coulomb force, as the slip is seen to continuously accelerate towards the terminal rupture speed (Figure 6b,c). This behavior corresponds to the diminishing effect of the toughness compared to that of the Coulomb force-rate with increasing run-out distance, and bulk slip rate $V_{\text {eff }}$ approaching the zero toughness limit given in (25). Corresponding, zero-toughness reduction of the EoM can be solved for the normalized value of the terminal rupture speed

$$
v_{r, \lim }=\frac{\dot{\Delta T}}{\pi}\left[(a / b-1) \ln V_{\mathrm{eff}}^{\left(K_{c}=0\right)}-\Delta f_{o} / b\right]^{-1} \quad \text { with } \quad V_{\mathrm{eff}}^{\left(K_{c}=0\right)}=0.4569 \dot{\Delta T}
$$

shown by dashed black lines on Figure $6 \mathrm{~b}, \mathrm{c}$. 


\section{friction rate dependence $\boldsymbol{a} / \boldsymbol{b}$}
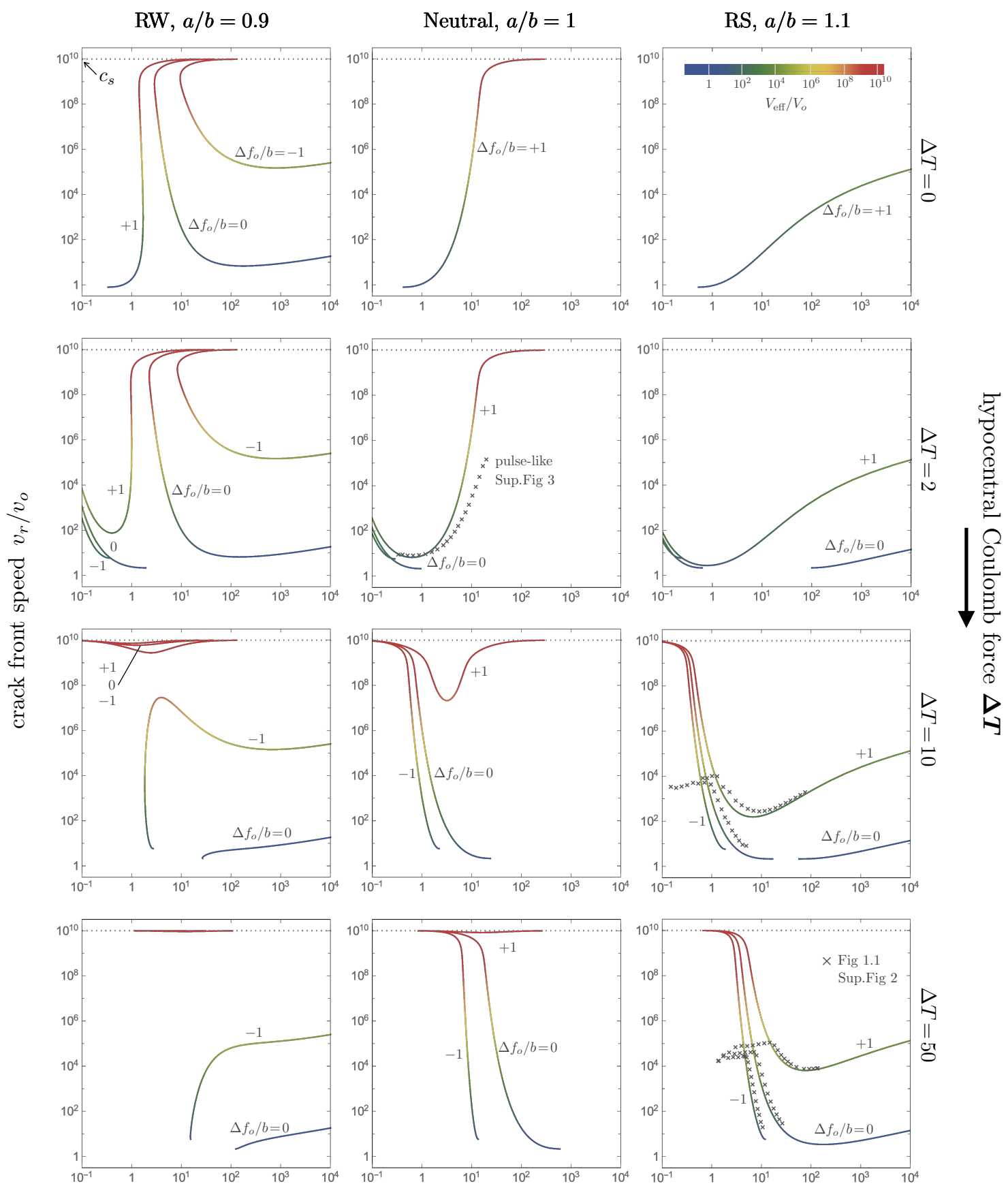

crack half-length $\ell / \ell_{b}$

Figure 5: Equation of motion for constant Coulomb hypocentral force - phase plot of the rupture front speed $v_{r}$ (scaled by $v_{o}=(\mu / b \bar{\sigma}) V_{o}$ ) vs. half-rupture-length $\ell$ (scaled by $\ell_{b}=(\mu / b \bar{\sigma}) L$ ) for a symmetric rupture on a fault with rate-state (slip law) friction characterized by rate-weakening (RW, left), rate-neutral (RN, middle), and rate-strengthening (RS, right) steady-state behaviour, and different initial overstress $\Delta f_{o} / b=-1,0,+1$. Top to bottom panels correspond to different values of Coulomb force $\Delta T / \mu L=0$ (top), 2, 10, and 50 (bottom). The effective slip rate magnitude within the bulk of the slipping patch is shown by color gradient. Several examples of $v_{r}$ vs. $\ell$ evolution in full numerical solutions of fault slip transients driven by localized pore overpressure due to the fixed volume injection (such as in Figure 1.1) are shown by ' $x$ ' for comparison. 

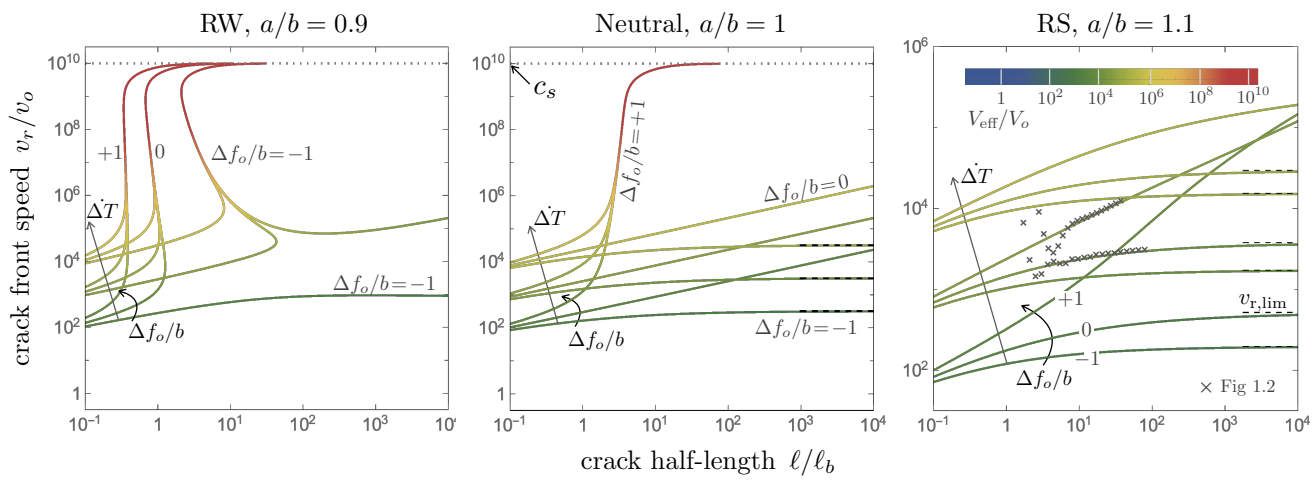

Figure 6: Equation of motion (31) for constant rate of Coulomb hypocentral force - phase plot of $v_{r}$ vs. $\ell$ for a symmetric rupture on a fault with rate-state (slip law) friction characterized by rate-weakening (left), rate-neutral (middle), and rate-strengthening (right) behaviour, various initial overstress $\Delta f_{o} / b=-1,0,+1$, and various values of the Coulomb force rate $\Delta T / \mu V_{o}=$ $10^{3}, 10^{4}, 10^{5}$. The effective slip rate magnitude within the bulk of the slipping patch is shown by color gradient. Terminal (large run-out) rupture velocity $v_{\mathrm{r}, \mathrm{lim}}$ for the rate neutral (middle panel) and strengthening (right panel) friction is shown by horizontal dashed lines.

\section{Implications for slip induced by fluid injection}

The EoM can be applied to study the fluid-injection induced slip, e.g., in the context of the two Scenarios discussed in the Introduction and Figure 1, provided that the hypocentral Coulomb force (21) and its evolution can be evaluated based on the history of fluid injection and its associated pore pressure perturbation on the fault. In the absence of the shear stress perturbation, $\Delta T_{b g}=0$, the Coulomb force is $\Delta T(t)=f_{0} \int \Delta p(z, t) d z$ where the pore pressure perturbation is approximately confined to a hypocentral region along the fault, $|z|<\ell_{0}(t)$, with half-length $\ell_{0}(t)$.

In the injection scenario 1 (Figure 1.1), when injecting into a hydraulically sealed reservoir abated by a fault, $2 \ell_{0}=h=$ the thickness of the reservoir, while the pore pressure perturbation is proportional to the injected fluid volume $V_{\mathrm{inj}}, \Delta p=V_{\mathrm{inj}} / \beta V_{\text {reservoir }}$, [23], where $\beta$ is the pore compressibility of the reservoir rock, thus, yielding, $\Delta T=f_{0} V_{\text {inj }} /\left(\beta V_{\text {reservoir }} / h\right)[\mathrm{N} / \mathrm{m}]$.

In the injection scenario 2 (Figure 1.2), when injecting directly into or nearby the permeable fault zone, $\ell_{0}=\sqrt{4 \alpha t}=$ pore pressure diffusion front run-out along the 1D-fault, and pore pressure can be found by convolution $\Delta p(z, t)=\int_{0}^{t}\left(d q / d t^{\prime}\right) p_{G}\left(z, t-t^{\prime}\right) d t^{\prime}$ of the injection rate $q(t)=\left(d V_{\mathrm{inj}} / d t\right) /\left(w_{\mathrm{FZ}} L_{\mathrm{inj}}\right)[\mathrm{m} / \mathrm{s}]$ (per unit permeable fault zone thickness $w_{F Z}$ and unit out-of-plane length of the fluid line-source, a segment of a horizontal injection wellbore parallel to the fault plane, $\left.L_{\mathrm{inj}}\right)$ with the Green's function given by the $q=1$ solution[36], $p_{G}(z, t)=(2 \sqrt{\pi} \alpha \beta)^{-1} \ell_{0}(t) \Pi\left(|z| / \ell_{0}(t)\right)$ and $\Pi(\xi)=\exp \left(-\xi^{2}\right)-\sqrt{\pi} \xi \operatorname{erfc}(\xi)$. Integrating the pore pressure perturbation over the fault, one gets a very useful result that the Coulomb force is simply proportional to the currently injected fluid volume, $\Delta T(t)=f_{0} V_{\mathrm{inj}}(t) /\left(\beta w_{\mathrm{FZ}} L_{\mathrm{inj}}\right)[\mathrm{N} / \mathrm{m}]$, irrespective of the injection history. Furthermore, this result is not restricted to the 1-D geometry of the problem, such that, e.g., for fluid injection into a $2 \mathrm{D}$-fault one finds $\Delta T(t)=f_{0} V_{\mathrm{inj}}(t) /\left(\beta w_{\mathrm{FZ}}\right)[\mathrm{N}]$.

The full numerical solution of slip transients in the two scenarios corresponding to fixed-volume (stopped) and fixed-volume-rate (continuing) injection are given in Figure 1 for the case of zero ambient overstress $\Delta f_{o}=0$ and the choice of injection parameters for which, in scaling (28), the 
Coulomb force $\Delta T \approx 50$ and the Coulomb-force-rate $\Delta T \approx 10^{4}$, respectively. When evaluating $\Delta T$ in these and other examples, we approximated the hypocentral friction $f_{0}=f_{s s}\left(V_{\text {eff }, 0}\right)$ by a representative constant ${ }^{15}$, which particular value has been chosen as the mean $f_{o}-\Delta f_{o} / 2$ of the background stress-ratio value $f_{o}$ and the steady-state friction value at the ambient slip rate $f_{\mathrm{ss}}\left(V_{o}\right)=f_{o}-\Delta f_{o}$. (We note though that this approximation likely breaks down in case of an earthquake slip instability, manifested by slip rate changes of many orders of magnitude, in which case rigorous evaluation of $f_{0}=f_{s s}\left(V_{\text {eff }, 0}\right)$ will be likely required, see Sup. Materials). The numerical rupture dynamics in the two scenarios (see Figure 1d, $\mathrm{d}^{\prime}$ for the rupture half-length vs. time) is contrasted to the EoM predictions in the phase plot of the rupture speed vs. rupture halflength in Figures 5 (bottom-right) and Figure 6c, respectively, where the numerical solutions are shown by ' $x$ '. We observe that EoM provides generally very good approximation of the decelerating (fixed-fluid-volume) and continuously accelerating (fixed-fluid-volume-rate) rupture dynamics in these two scenarios once the rupture is grown large enough compared to its hypocentral region. The EoM is seen to somewhat underpredict the rupture velocity in the fixed-volume case, which is attributed to the influence of the direct friction effect which tends to slightly reduce the friction in bulk of a decelerating rupture when compared to the its steady-state value (see Sup. Figure 8) and, thus, 'ease' the rupture propagation compared to the EoM prediction, where the direct effect is neglected in the bulk of the crack.

The EoM also provides a good approximation of the rupture dynamics (Figures 5,6) when injecting into initially overstressed $\left(\Delta f_{o}>0\right)$, rate-strengthening faults (see Sup. Figure 2 for the full numerical solutions). The only attempted numerical example which the EoM failed to approximate corresponds to the accelerating aseismic rupture on overstressed $\left(\Delta f_{o} / b=1\right)$, rateneutral fault $(a / b=1)$ with slip-law friction subject to perturbative force $\Delta T=2$ (Figures 5 ), which propagates as a slip pulse rather than a crack (see Figure 3a). The pulse-like propagation mode has been previously observed on rate-weakening, slip-law faults by [10]. When considering the same injection scenario and loading conditions, but on the ageing-law fault, the propagation is crack-like (Figure $3 \mathrm{a}^{\prime}$ ) and the EoM prediction is adequate (Sup. Figure 5).

\section{Conclusions}

In this work, we have developed a universal solution near the leading edge of a slip transient propagating on a fault with rate-state dependent friction. This 'tip solution' quantifies evolution of the slip velocity associated with the passage of the rupture front, characterized by the initial dramatic slip acceleration followed by deceleration towards the steady-state sliding in the bulk of the slipping patch. The corresponding maximum values of the slip velocity and shear stress perturbation carried by the rupture front (the latter related to the maximum excursion $\Delta f_{p}$ of friction from the steady-state) are established as functions of the instantaneous rupture front velocity $v_{r}$. The characteristic size $\ell_{c}\left(v_{r}\right)$ of this near tip process zone, shown to be a small fraction (e.g., given by $b / \Delta f_{p}\left(v_{r}\right)$ for the slip-law of the rate-state friction) of the classical frictional lengthscale $\ell_{b}=(\mu / b \bar{\sigma}) L$, provides an upper bound for the fault discretization size necessary to resolve slip transients by numerical approaches $[15,19]$.

Developed tip solution allows for explicit evaluation of the fracture energy $G_{c}\left(v_{r}\right)$ dissipated within the near tip process zone when overcoming the frictional resistance $\Delta f$ in excess of the steady-state value. This opens a possibility to model slip transients on rate-state faults as singular

\footnotetext{
${ }^{15}$ in lieu of the weak dependence of the steady-state friction $f_{\mathrm{ss}}$ on $V$, which typically amounts to a percent or less change in $f_{\mathrm{ss}}$ over few orders of magnitude change of $V$
} 
cracks characterized by the simplified, steady-state frictional resistance in the bulk of the fracture, and by a stress singularity at the fracture tip which intensity is given by $G_{c}\left(v_{r}\right)$. We have pursued this route and developed an analytical equation of motion for 1D slip transients driven by a combination of an uniformly-distributed background overstress $\Delta f_{o}$ and a localized fault strength perturbation at the hypocentral region, quantified by the net Coulomb force $\Delta T(t)$.

We have studied general properties of slip transients predicted by the equation of motion and further validated by a number of fully numerical slip simulations. Particularly, we have established that the maximum run-out distance of an aseismic slip transient on a rate-strengthening fault is $\propto \Delta T^{2}$ when the Coulomb force $\Delta T$ is a constant $(\Delta T=0)$, and that the long-term rupture speed $v_{r, \lim }$ of the transients is $\propto \Delta T$ when $\Delta T>0$. In the context of slip induced by fluid injection, the hypocentral Coulomb force is shown to be proportional to the injected fluid volume, e.g., $\Delta T(t)=f_{0} V_{\mathrm{inj}}(t) / \beta w_{\mathrm{FZ}} L_{\mathrm{inj}}$ in the case of injection into the permeable/damaged fault zone of thickness $w_{\mathrm{FZ}}$ over injection interval length $L_{\mathrm{inj}}$, where $\beta$ is the pore compressibility of the fault rock and $f_{0}$ a representative value of fault friction. The EoM solution then provides (i) a time-dependent upper bound for aseismic slip propagation on a frictionally-stable fault $\ell_{\lim }(t) \sim \int v_{r, \lim } d t \propto V_{\text {inj }}(t)$ while the injection is ongoing, and (ii) the maximum run-out of the induced slip, $\ell_{\max } \propto V_{\text {inj,total }}^{2}$, once injection has been stopped.

Developed slip equation of motion and its possible extension to 2D slip, provide a relatively simple analytical tool which can be applied to study a variety of transient fault slip phenomena, slow and fast, natural and induced by anthropogenic activities.

Acknowledgements: D.I.G. would like to acknowledge support for this research by the Natural Science and Engineering Research Council of Canada (Discovery grant 05743).

\section{References}

[1] C. H. Scholz. The mechanics of earthquakes and faulting. Cambridge University Press, 2nd edition, 2002.

[2] T. Baumberger and C. Caroli. Solid friction from stick-slip down to pinning and aging. Adv. Phys., 55:279-348, 2006.

[3] J.H. Dieterich. Modeling of rock friction 1. experimental results and constitutive equations. J. Geophys. Res., 84(15):2161-2168, 1979.

[4] A. Ruina. Slip instability and state variable friction laws. J. Geophys. Res., 88(B12):1035910370, 1983.

[5] C. Marone. Laboratory-derived friction laws and their application to seismic faulting. Annu. Rev. Earth Planet. Sci., 26:643-696, 1998.

[6] J. R. Rice. Heating and weakening of faults during earthquake slip. J. Geophys. Res., 111:B05311, 2006.

[7] R. C. Viesca and D. I. Garagash. Ubiquitous weakening of faults due to thermal pressurization. Nature Geoscience, 8:875-879, 2015.

[8] G. Di Toro, R. Han, T. Hirose, N. De Paola, S. Nielsen, K. Mizoguchi, F. Ferri, M. Cocco, and T. Shimamoto. Fault lubrication during earthquakes. Nature, 471:494-498, 2011. 
[9] A. M. Rubin and J.-P. Ampuero. Earthquake nucleation on (aging) rate and state faults. $J$. Geophys. Res., 110:B11312, 2005.

[10] J.-P. Ampuero and A. M. Rubin. Earthquake nucleation on rate and state faults: Aging and slip laws. J. Geophys. Res., 113:B01302, 2008.

[11] R. Viesca. Self-similar slip instability on interfaces with rate- and state-dependent friction. Proc. Roy. Soc. London Ser. A, 472:20160254, 2016.

[12] G. Perrin, J. R. Rice, and G. Zheng. Self-healing slip pulse on a frictional surface. J. Mech. Phys. Solids, 43:1461-1495, 1995.

[13] H. Noda, E. M. Dunham, and J. R. Rice. Earthquake ruptures with thermal weakening and the operation of major faults at low overall stress levels. J. Geophys. Res., 114:B07302, 2009.

[14] A.-A. Gabriel, J.-P. Ampuero, L. A. Dalguer, and P. M. Mai. The transition of dynamic rupture styles in elastic media under velocity-weakening friction. J. Geophys. Res., 117:B09311, 2012.

[15] J. R. Rice. Spatio-temporal complexity of slip on a fault. J. Geophys. Res., 98(B6):9885-9907, 1993.

[16] N. Lapusta, J. R. Rice, Y. Ben-Zion, and G. Zheng. Elastodynamic analysis for slow tectonic loading with spon- taneous rupture episodes on faults with rate- and state- dependent friction. J. Geophys. Res., 105(B10):23,765-23,789, 2000.

[17] Y. Liu and J. R. Rice. Spontaneous and triggered aseismic deformation transients in a subduction fault model. J. Geophys. Res., 112:B09404, 2007.

[18] P. A. Segall, A. M. Rubin, A. M. Bradley, and J. R. Rice. Dilatant strengthening as a mechanism for slow slip events. J. Geophys. Res., 115:B12305, 2010.

[19] H. Perfettini and J.-P. Ampuero. Dynamics of a velocity strengthening fault region: Implications for slow earthquakes and postseismic slip. J. Geophys. Res., 113:B09411, 2008.

$[20]$ T. S. Eyre, D. W. Eaton, D. I. Garagash, M. Zecevic, M. Venieri, R. Weir, and D. C. Lawton. The role of aseismic slip in hydraulic fracturing-induced seismicity. Sci. Adv., 5:eaav7172, 2019.

[21] P. Dublanchet. Fluid driven shear cracks on a strengthening rate-and-state frictional fault. $J$. Mech. Phys. Solids, 132:103672, 2019.

[22] I. Svetlizky, E. Bayart, and J. Fineberg. Brittle fracture theory describes the onset of frictional motion. Ann. Rev. Condens. Matter Phys., 10:253-273, 2019.

[23] M. Galis, J.-P. Ampuero, P. M. Mai, and F. Cappa. Induced seismicity provides insight into why earthquake ruptures stop. Sci. Adv., 3:eaap7528, 2017.

[24] D. I. Garagash and L. N. Germanovich. Nucleation and arrest of dynamic slip on a pressurized fault. J. Geophys. Res., 117:B10310, 2012.

[25] J. R. Rice. Constitutive relations for fault slip and earthquake instabilities. Pure Appl. Geophys., 121(3):443-475, 1983. 
[26] J. R. Rice. Mathematical analysis in the mechanics of fracture. In H. Liebowitz, editor, Fracture, an Advanced Treatise, volume II, chapter 3, pages 191-311. Academic Press, New York NY, 1968.

[27] F. Barras, M. Aldam, T. Roch, E. A. Brener, E. Bouchbinder, and J.-F. Molinari. The emergence of crack-like behavior of frictional rupture: Edge singularity and energy balance. Earth ES Plan. Sci. Lett., 531:115978, 2020.

[28] D. I. Garagash. Seismic and aseismic slip pulses driven by thermal pressurization of pore fluid. J. Geophys. Res., 117:B04314, 2012.

[29] F. Cappa, M. M. Scuderi, C. Collettini, Y. Guglielmi, and J.-P. Avouac. Stabilization of fault slip by fluid injection in the laboratory and in situ. Sci. Adv., 5:eaau4065, 2019.

[30] P. Bhattacharya and R. C. Viesca. Fluid-induced aseismic fault slip outpaces pore-fluid migration. Science, 364:464-468, 2019.

[31] T.E. Tullis. Rock friction constitutive behavior from laboratory experiments and its implications for an earthquake field monitoring program. Pure Appl. Geophys., 126:555-588, 1988.

[32] L. B. Freund. Dynamic Fracture Mechanics. Cambridge University Press, Cambridge, 1990.

[33] M. Abramowitz and I. A. Stegun, editors. Handbook of Mathematical Functions. Dover, 1965.

[34] R. C. Viesca and D. I. Garagash. Numerical methods for coupled fracture problems. J. Mech. Phys. Solids, 113:13-34, 2018.

[35] K. B. Broberg. Cracks and Fracture. Academic Press, 1999.

[36] H. Carslaw and J. C. Jaeger. Conduction of Heat in Solids. Oxford University Press, 2nd edition, 1959. 


\section{A Supplementary Material}

\section{A.1 Small scale yielding rational for rate-state fault ruptures}

The above treatment of the near rupture tip region suggests that the way for approximate s.s.y. modeling of the rupture propagation, when the rupture process is such that the frictional evolution away from the frictional steady-state is mostly confined to the region adjacent to the advancing front, while the friction is approximately at the steady-state within the rest of the slipping patch, as previously suggested by [25]. If so, the rupture can be modeled by that overcoming the steadystate frictional fault strength in the bulk of the slipping region and the finite fracture energy at the advancing front.

Consider therefore the 'outer' solution for the slipping patch sliding at the steady-state fault friction $\tau^{\text {out }}(x)=f_{s s}\left(V^{\text {out }}(x)\right) \bar{\sigma}$ valid in the bulk of the crack, i.e. away from the tip $x \gg \ell_{c}$, where $x=\ell-|X|$ denotes the distance from the crack tip, and $\ell_{c}$ is the small spatial scale of the tip process zone, $\ell_{c} \ll \ell$. While within the fracture tip process zone, $x \ll \ell$, the corresponding 'tip' solution $\tau^{t i p}(x)=f^{t i p}(x) \bar{\sigma}$ is to describe the away-from-steady-state slip there. For such a composite solution structure to be realized, the two solutions have to match over the intermediate distances from the tip $\ell_{c} \ll x \ll \ell$, where both solutions reduce to the intermediate asymptote corresponding to the LEFM solution with a finite fracture energy $G_{c}$,

$$
\ell_{c} \ll x \ll \ell: \quad V^{\text {out }}(x) \approx V^{\text {tip }}(x) \approx V^{\mathrm{LEFM}}(x)=V_{c} \frac{2}{\sqrt{\pi x / \ell_{c}}}
$$

(The slip $V_{c}$ rate and distance $\ell_{c}$ scales defined in the main text in terms of $G_{c}$. With this definitions the LEFM asymptote takes a familiar form $V^{\mathrm{LEFM}} / v_{r}=(d \delta / d x)^{\mathrm{LEFM}}=\sqrt{2 G_{c} / \pi \bar{\mu} x}$.

\section{A.2 The rupture tip (process zone) solution}

Let us henthforth focus on the tip solution and drop the subscript tip for brevity. In view of the above, the inner solution has to satisfy the equation of elastic shear stress transfer, modeled by that of a semi-infinite crack with background stress-level corresponding to the intermediate (LEFM) stress asymptote

$$
f(x)-f_{s s}\left(V^{\mathrm{LEFM}}(x)\right)=\frac{\bar{\mu}\left(v_{r}\right)}{2 \pi \bar{\sigma}} \int_{0}^{\infty} \frac{d \delta / d x^{\prime}}{x^{\prime}-x} d x^{\prime}, \quad V=v_{r} \frac{d \delta}{d x},
$$

and by the reduced form of the friction law within the tip process zone, as given by the slipweakening relation (9) for the friction departure from the steady-state $\Delta f=f-f_{s s}(V)$

$$
\Delta f=\Delta f(\delta)
$$

In view of the above, the stress transfer equation along the crack can then be rewritten

$$
\Delta f(\delta(x))+\left[f_{s s}(V(x))-f_{s s}\left(V^{\mathrm{LEFM}}(x)\right)\right]=\frac{\bar{\mu}\left(v_{r}\right)}{2 \pi \bar{\sigma}} \int_{0}^{\infty} \frac{d \delta / d x^{\prime}}{x^{\prime}-x} d x^{\prime}
$$

and the fracture energy as

$$
G_{c} / \bar{\sigma}=\int_{0}^{\infty}\left(f(x)-f_{s s}\left(V^{\mathrm{LEFM}}(x)\right)\right) d \delta(x)=\int_{0}^{\infty} \Delta f(\delta) d \delta+\int_{0}^{\infty}\left[f_{s s}(V(x))-f_{s s}\left(V^{\mathrm{LEFM}}(x)\right)\right] d \delta(x)
$$


In solving the inner problem formulated in the above, we further neglect the bracketed terms under assumption that the variation of the steady-state value of friction in the process zone from its far-field (LEFM) value is small compared to the friction departure from the steady-state, i.e. $f_{s s}(V)-f_{s s}\left(V^{\text {LEFM }}\right) \ll f-f_{s s}(V)$. The tip solution under the above assumptions is given in the main text.

Consider estimating the goodness of the approximations taken in deriving the sharp-tip rupture solution and associated fracture energy $G_{c}$. The approximations were twofold.

Firstly, the direct friction effect has been neglected in the near tip friction evolution. While the assumption is plausible when friction undergoes relatively large changes driven by the evolution of the state (i.e. away from the steady-state), it is expected to break down in the far field of the tip solution, as the latter approaches the steady state $\left(\Delta f \rightarrow 0\right.$ as $\left.x / \ell_{c} \rightarrow \infty\right)$. To evaluate the associated error, we use the obtained tip solution for the slip $\delta$ and slip rate $V$ to integrate the full rate-state friction equation (6), and compare the result, denoted as $\Delta f^{\text {r-s }}$, to the tip solution value $\Delta f$ (see Figure 7a for the case of slip law rate-state friction solution). The corresponding relative error associated with the use of the approximate fracture energy $G_{c}=\bar{\sigma} \int_{0}^{\infty} \Delta f(\delta) d \delta=\Delta f_{p} \bar{\sigma} \delta_{c}$ can be defined as

$$
\mathscr{E}^{\mathrm{r}-\mathrm{s}}\left(G_{c}\right)=\frac{1}{G_{c}} \bar{\sigma} \int_{0}^{\infty}\left[\Delta f^{\mathrm{r}-\mathrm{s}}(\delta)-\Delta f(\delta)\right] d \delta
$$

It can be seen from Figure 7a, that the direct effect slightly modifies how the friction change $\Delta f$ vanishes at large slip, specifically, $\Delta f^{\text {r-s }}$ is seen to dip below zero before returning asymptotically to zero as $\sim-\delta_{c} / \delta$ at large slip (the feature observed before in the rupture tip solution accounting for direct effect [Viesca\&Garagash, 2012/3?). As the result, the integral in $\mathscr{E}^{\text {r-s }}\left(G_{c}\right)$ is actually diverging logarithmically with large slip. We are not that interested in (or discouraged by :) this logarithmic "tail" of the fracture energy at large slip (since most of the fault weakening is accomplished over slip distances $\sim \delta_{c}$ ), and replace the upper (infinite) limit of integration in (35) by the slip value at the $\min \Delta f^{\text {r-s }}$ in evaluating the fracture energy error $\mathscr{E}^{\mathscr{r}-\mathrm{s}}\left(G_{c}\right)$. The resulting error estimate is plotted as function of $\Delta f_{p} / b$ on Figure $7 \mathrm{~b}$ for the slip law rate-state friction case.

Secondly, the bracketed term in (33) and (34), corresponding to the variability of the steadystate friction value within the process zone, has been neglected in the near tip solution. To evaluate the validity of this assumption we use the tip solution to evaluate the bracketed term contribution to the full expression for the fracture energy (34), and defining the relative error associated with the approximate fracture energy $G_{c}=\Delta f_{p} \bar{\sigma} \delta_{c}$ as,

$\mathscr{E}\left(G_{c}\right)=\frac{1}{G_{c}} \bar{\sigma} \int_{0}^{\infty}\left[f_{s s}(V(x))-f_{s s}\left(V^{\mathrm{LEFM}}(x)\right)\right] d \delta(x)=\chi \frac{a-b}{\Delta f_{p}} \quad$ with $\quad \chi=\int_{0}^{\infty} \ln \left[\frac{V / V_{c}}{2 / \sqrt{\pi x / \ell_{c}}}\right] \frac{d \delta}{\delta_{c}}$

Prefactor $\chi=\chi\left(\Delta f_{p} / b\right)$ can be evaluated from the sharp-tip solution: it is a constant $\approx 0.816$ for the slip law rate-state friction, and is a function with values between $\chi(0) \approx 0.816$ and $\chi(\infty) \approx$ 0.936 for the ageing law. Error $\mathscr{E}\left(G_{c}\right)$ is shown as a function of $\Delta f_{p} / b$ by a dashed line in Figure $7 \mathrm{~b}$ for the slip law rate-state friction case with $a / b=1.1$.

\section{A.3 Hypocentral slip rate}

Considerations similar to those for the bulk effective slip rate lead to the following expression for the hypocentral effective slip rate 


$$
V_{\text {eff }, 0}=\mathscr{F}_{0}\left(\frac{1}{\mathscr{P}}-\ln \frac{\ell_{0}}{2 \ell}\right) \times \frac{2 \Delta \dot{T}}{\hat{\mu}} \quad \text { with } \quad \mathscr{F}_{0}(z)=z e^{-e^{z} \operatorname{Ei}(-z)}
$$

It can be established that $V_{\text {eff, }, 0}$ varies between the toughness-dominated $V_{\mathrm{eff}, 0}^{(\dot{*} T=0)}=2 \frac{K_{\mathrm{c}} \dot{\ell}}{\hat{\mu} \sqrt{\pi \ell}}$ (a half of the corresponding bulk value $(25))$ and the point-force-rate-dominated $V_{\text {eff }, 0}^{\left(K_{c}=0\right)}=\mathscr{F}_{0}\left(-\ln \frac{\ell_{0}}{2 \ell}\right) \frac{2 \Delta T}{\hat{\mu}}$ limits with increasing $\mathscr{P}$. Substituting (37) into $\Delta T=\Delta T_{b g}-f_{s s}\left(V_{\text {eff }, 0}\right) \Delta N$, (21), allows to solve for $\Delta T$. Consider a simplified approach to the solution, where we differentiate the latter with respect to time while assuming that $f_{s s}\left(V_{\text {eff }, 0}\right)$ is a slowly varying quantity, resulting in $\dot{\Delta T} \approx \Delta T_{b g}-f_{s s}\left(V_{\text {eff }, 0}\right) \Delta N$. The latter together with (37) provides implicit algebraic equation to solve for $\Delta T$ and $V_{\text {eff, }, 0}$ as function of $\ell$ and $\dot{\ell}$ (while extent $\ell_{0}$ of the hypocentral region and corresponding normal $\Delta N$ and shear $\Delta T_{b g}$ perturbative forces and their rates are known). 


\section{B Supplementary Figures}
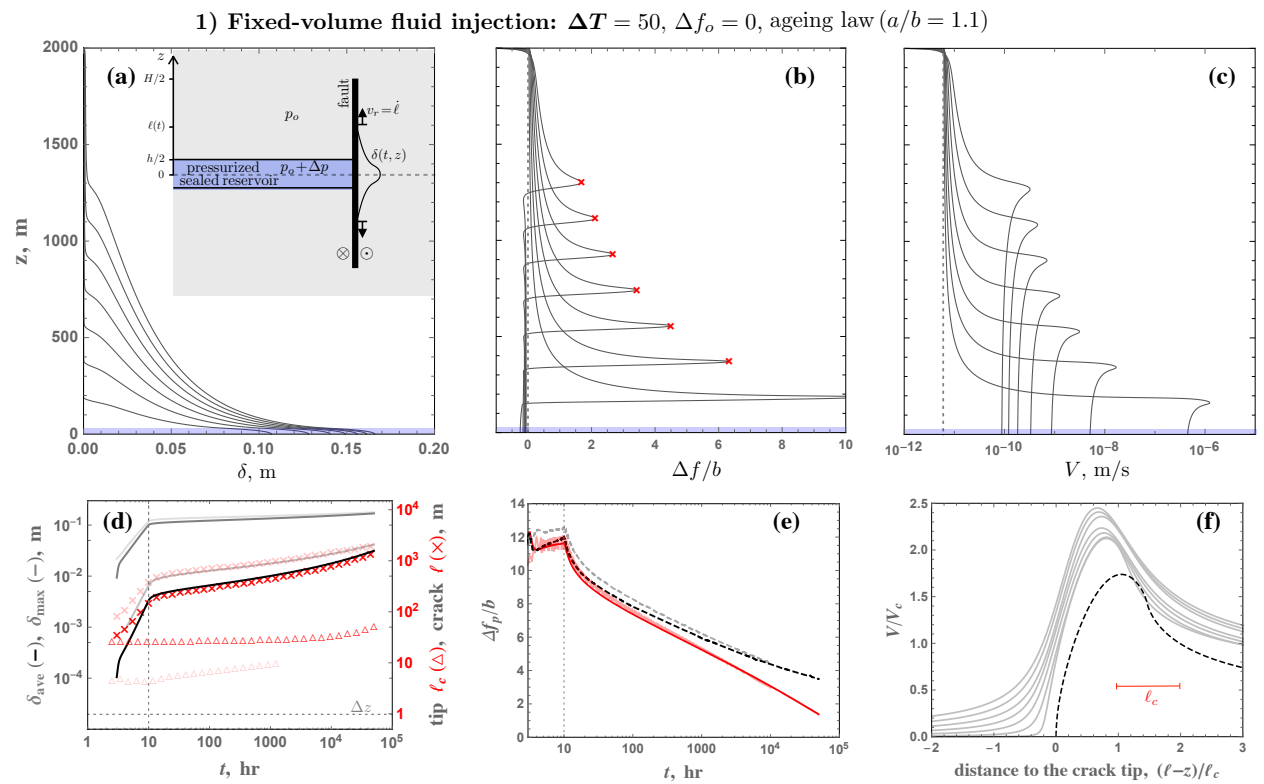

2) Fixed-volume-rate fluid injection: $\dot{\Delta T}=10^{4}, \Delta f_{o}=0$, ageing law $(a / b=1.1)$
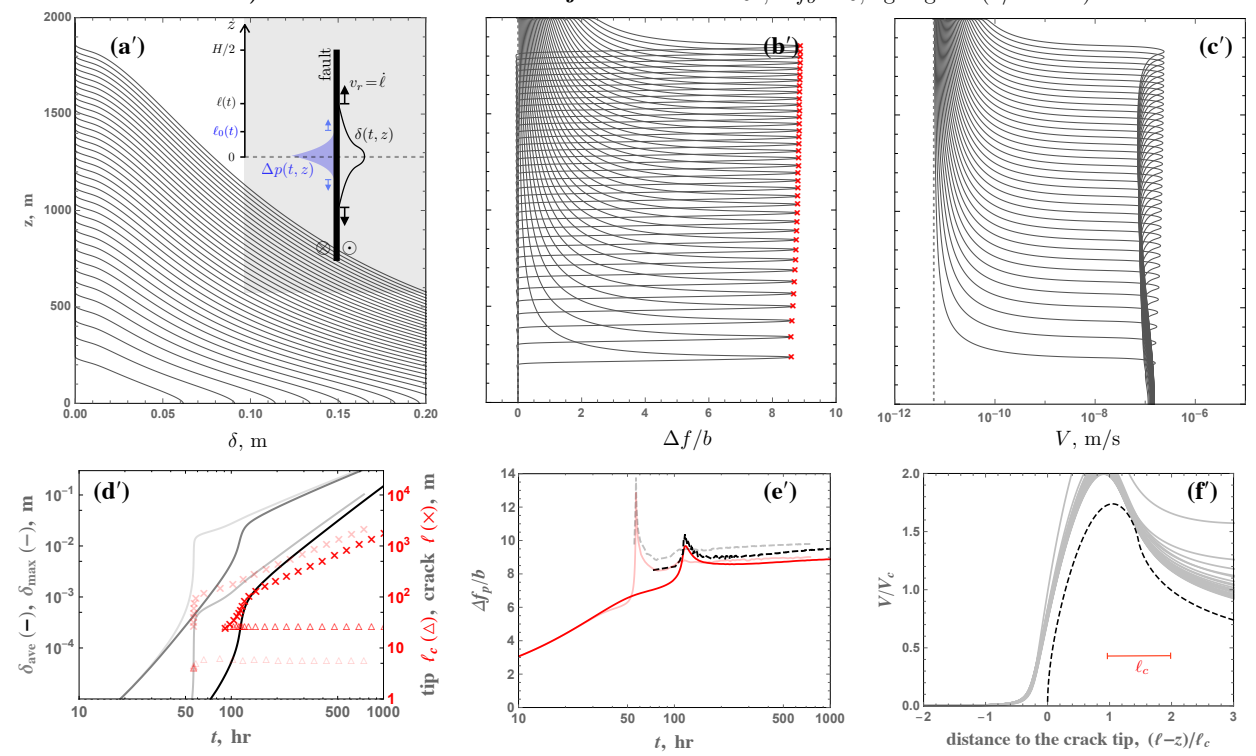

Figure 1: Symmetric slow slip development on a fault characterized by a rate-strengthening, ageinglaw friction for the injection scenarios 1 (a-f) and 2 (a'-f') and zero initial fault overstress. See Figure 1 for the same slip scenarios / fault loading, but different fault friction (slip law). The rupture length evolution and some other slip characteristics from Figure 1 are shown here in $(\mathrm{d}, \mathrm{e})$ and (d',e') by opaque symbols/lines for comparison. 

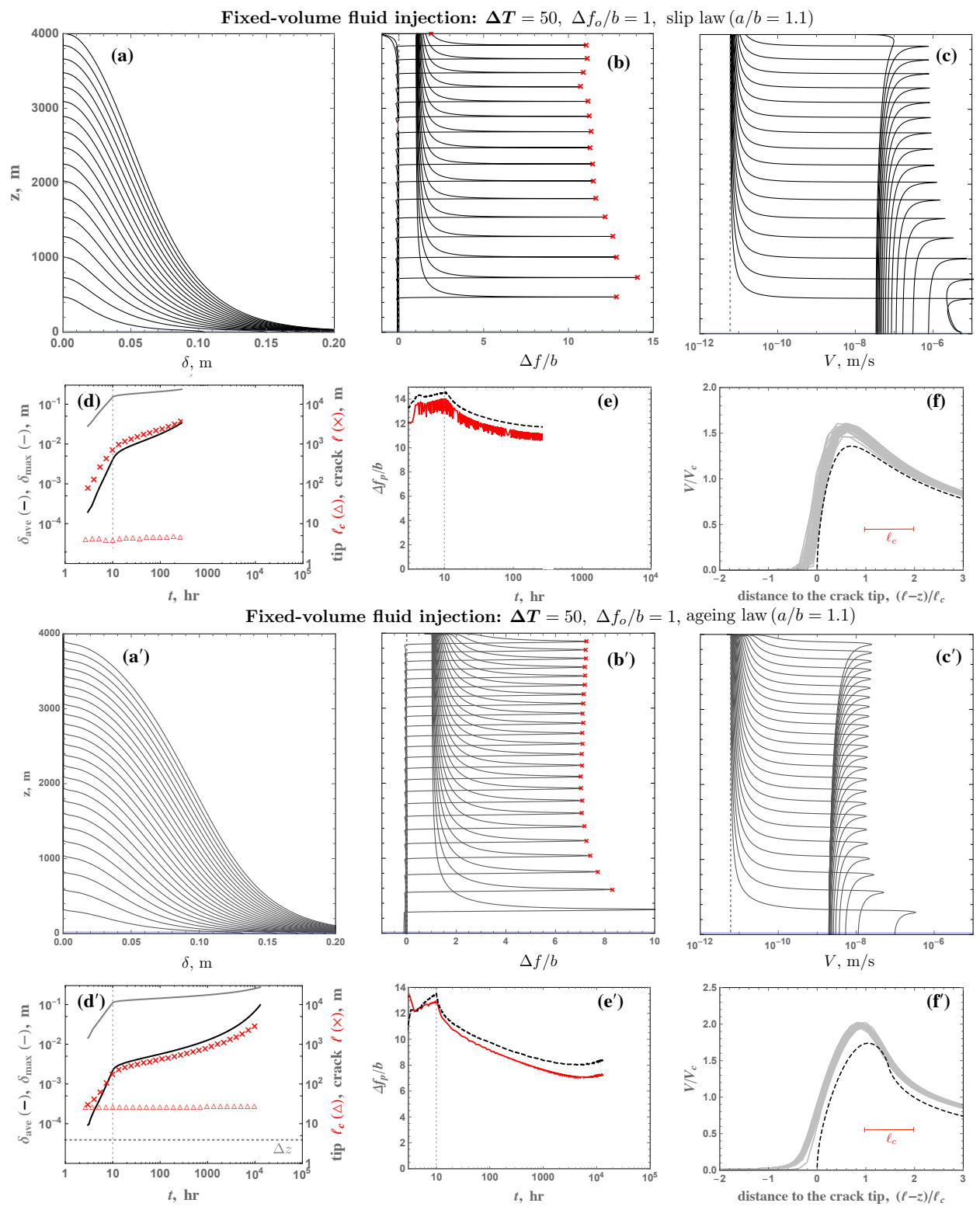

Figure 2: Symmetric slow slip development on a fault characterized by a rate-strengthening, sliplaw (a-f) and ageing-law (a'-f') friction for the injection scenario 1 and non-zero initial overstress. See Figure 1.1 and Sup. Figure 1.1 for the same slip scenarios bar different (zero) initial overstress. 

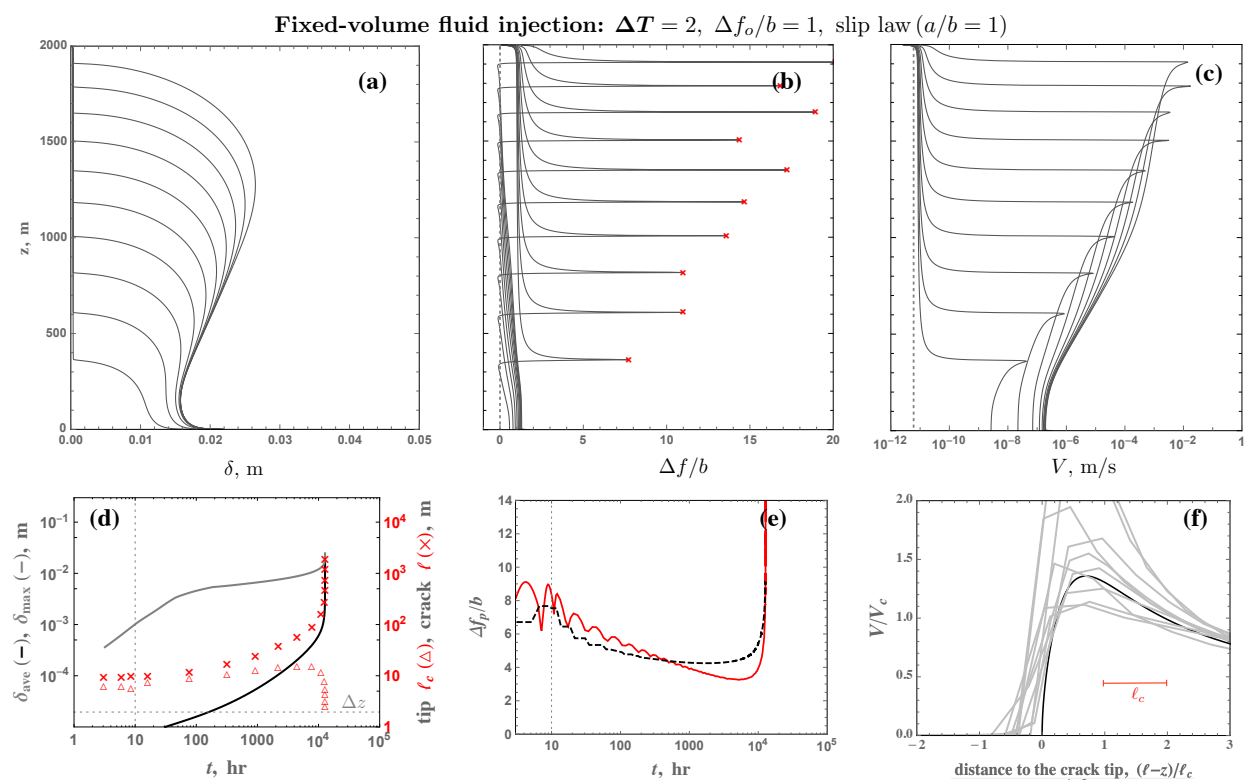

Fixed-volume fluid injection: $\boldsymbol{\Delta} \boldsymbol{T}=2, \Delta f_{o} / b=1$, ageing law $(a / b=1)$
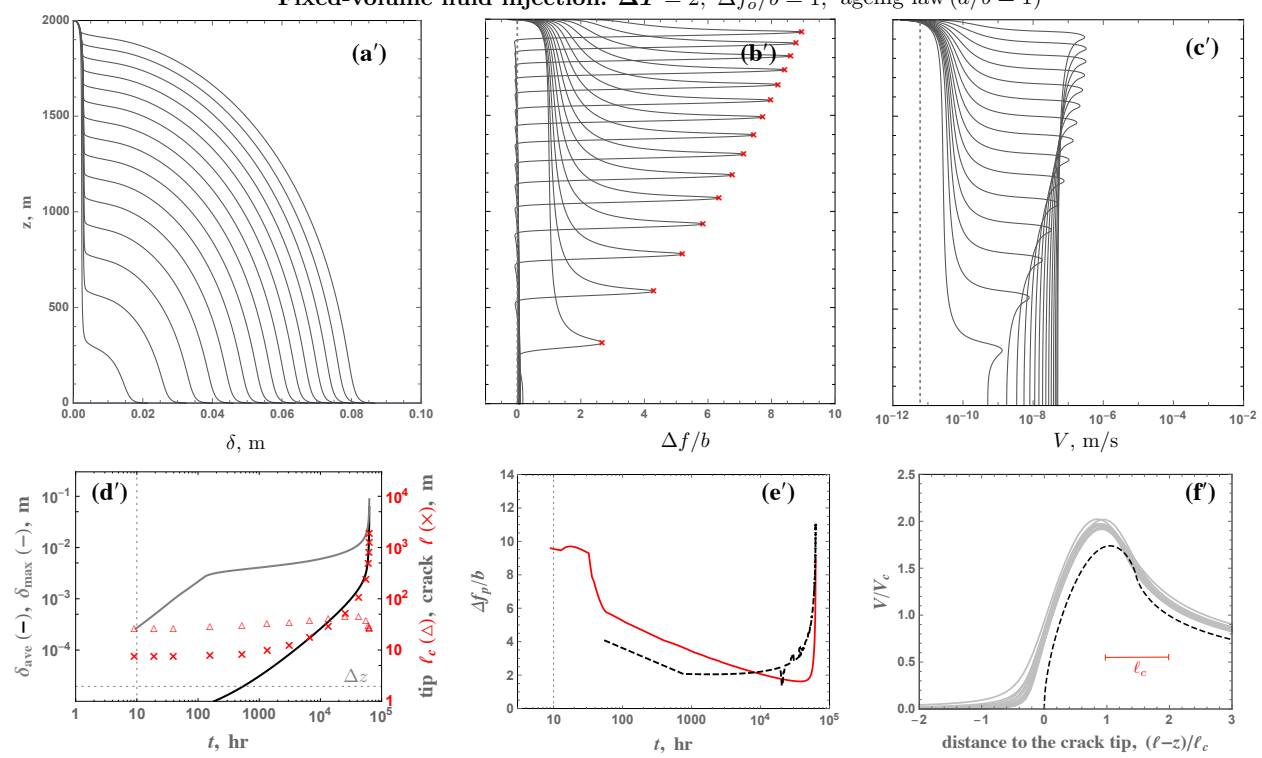

Figure 3: Symmetric slow slip development on a fault characterized by a rate-neutral, slip-law (a-f) and ageing-law (a'-f') friction for the injection scenario 1 and non-zero initial overstress. Note the pulse-like rupture mode (a) for the slip-law friction case, compared to the usual crack-like mode (a') for the ageing friction. (See Sup. Figure 2 for the same slip scenario and initial fault overstress conditions, but different sense of the friction rate-dependence (rate-strengthening), where rupture propagation is crack-like irrespective of the friction law). 


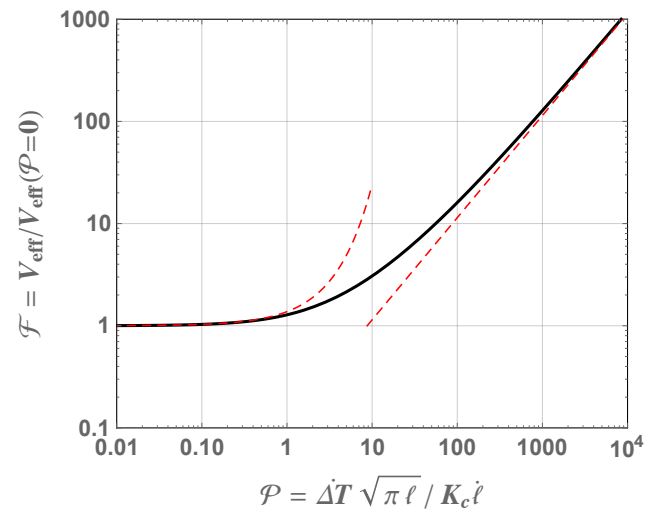

Figure 4: Normalized effective slip rate $\mathscr{F}$ as function of normalized point-load-rate $\mathscr{P},(27)$. The small/large $\mathscr{P}$ asymptotes (see the text) are shown by dashed lines. 


\section{friction rate dependence $\boldsymbol{a} / \boldsymbol{b}$}
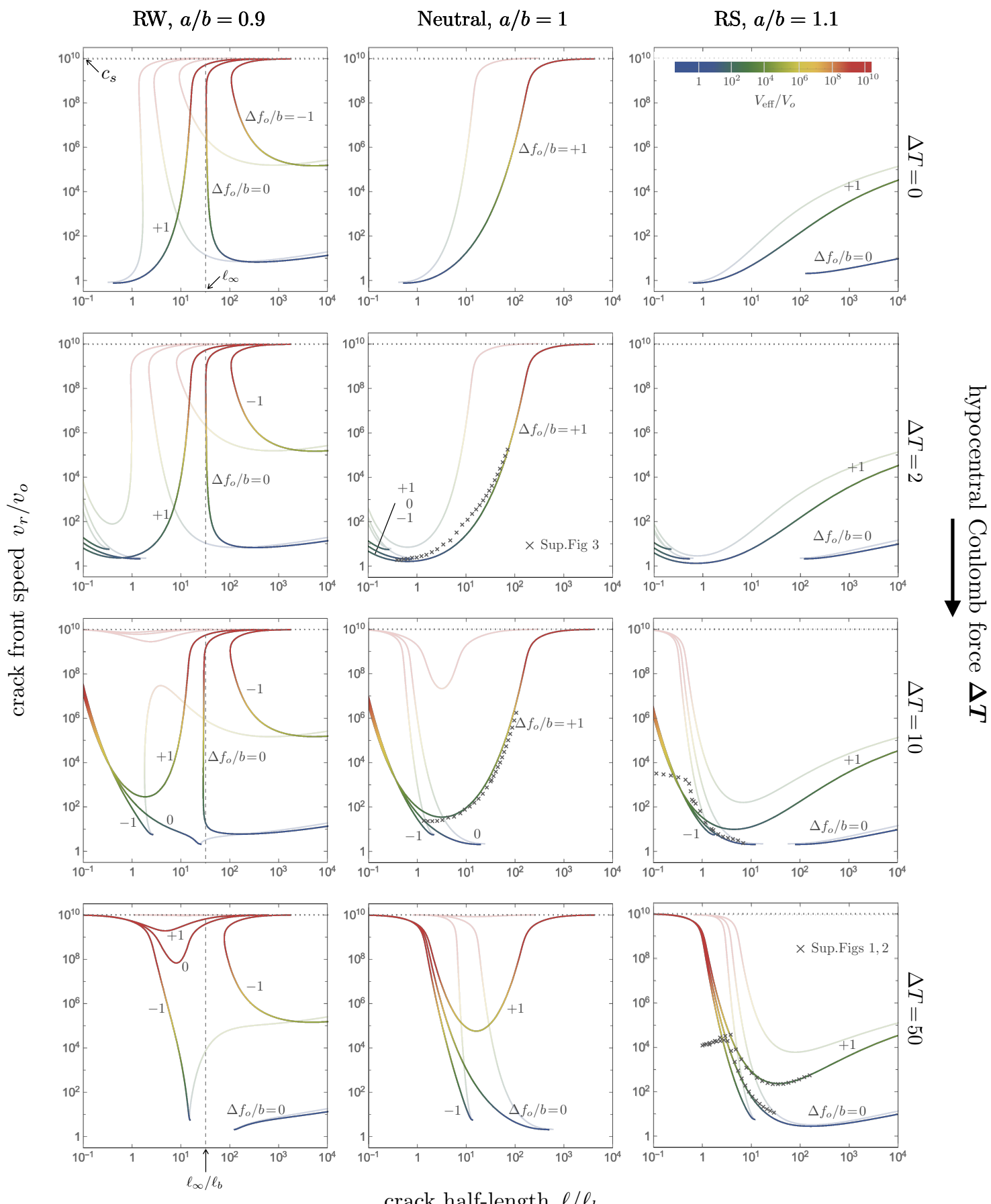

crack half-length $\ell / \ell_{b}$

Figure 5: Same as Figure 5 but for the ageing law of rate-state friction: Equation of motion for constant Coulomb hypocentral force - phase plot of the rupture front speed $v_{r}$ (scaled by $v_{o}=(\mu / b \bar{\sigma}) V_{o}$ ) vs. half-rupture-length $\ell$ (scaled by $\left.\ell_{b}=(\mu / b \bar{\sigma}) L\right)$ for a symmetric rupture on a fault with ratestate (ageing law) friction characterized by rate-weakening (left), rate-neutral (middle), and ratestrengthening (right) steady-state behaviour, and different initial overstress $\Delta f_{o} / b=-1,0,+1$. Top to bottom panels correspond to different values of the constant Coulomb hypocentral force $\Delta T /\left(b \sigma \ell_{b}\right)=0$ (top), 2,10 , and 50 (bottom). The effective slip rate magnitude within the bulk of the slipping patch is shown by color gradient. The characteristic lengthscale $\ell_{\infty}=\ell_{b} /\left(\pi(1-a / b)^{2}\right)$ is shown by vertical dashed line for the RW friction case (left panel). Phase plots for the slip law of rate-state friction of Figure 5 are shown by opaque curves for comparison. 

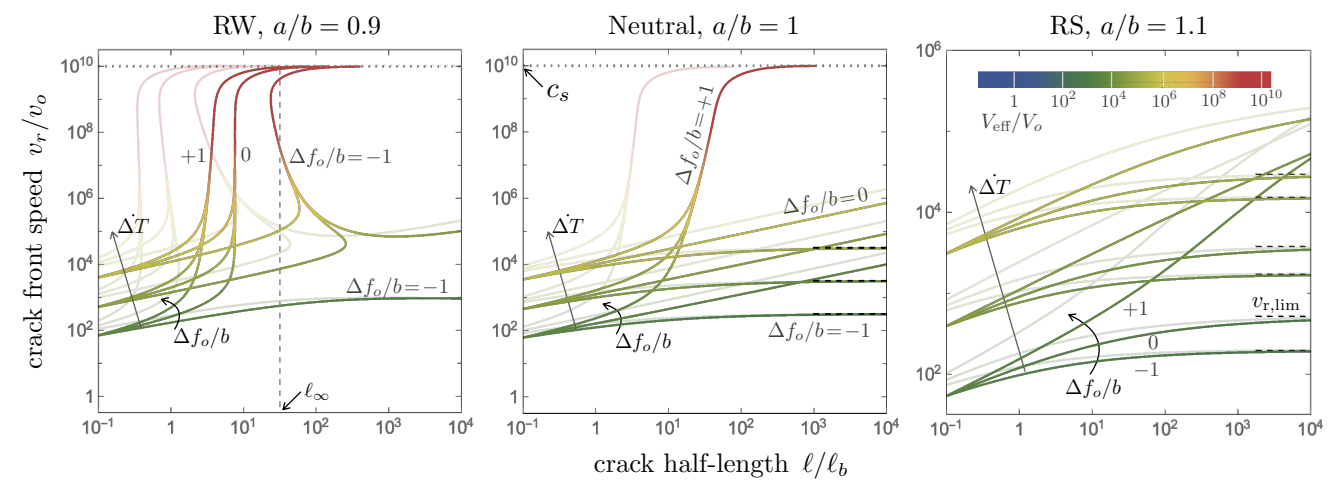

Figure 6: Same as Figure 6 but for the ageing law of rate-state friction: Equation of motion for constant rate of Coulomb hypocentral force - phase plot of $v_{r}$ vs. $\ell$ for a symmetric rupture on a fault with rate-state (slip law) friction characterized by rate-weakening (left), rate-neutral (middle), and rate-strengthening (right) behaviour, various initial overstress $\Delta f_{o} / b=-1,0,+1$, and various values of the Coulomb force rate $\Delta T / \mu V_{o}=10^{3}, 10^{4}, 10^{5}$. The effective slip rate magnitude within the bulk of the slipping patch is shown by color gradient. Terminal (large runout) rupture velocity $v_{\text {r,lim }}$ for the rate neutral (middle panel) and strengthening (right panel) friction is shown by horizontal dashed lines. Phase plots for the slip law of rate-state friction of Figure 6 are shown by opaque curves for comparison. Several examples of $v_{r}$ vs. $\ell$ evolution in full numerical solutions of fault slip transients driven by fixed volume-rate injection (such as in Figure $1.2)$ are shown by ' $x$ ' for comparison.
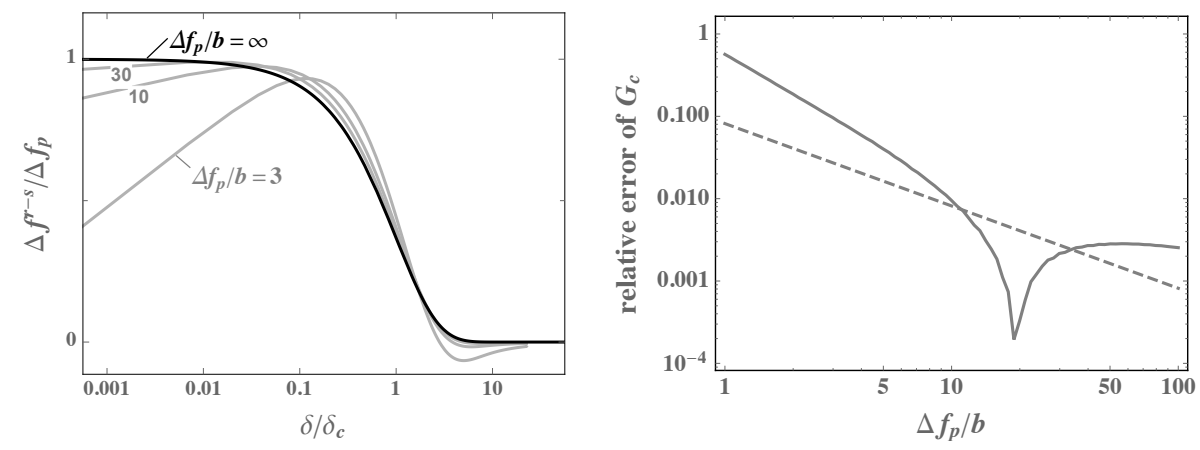

Figure 7: (a) Normalized friction departure from the steady-state $\Delta f^{r-s} / \Delta f^{p}$ vs. slip $\delta / \delta_{c}$ obtained from integration of the full form of the slip law rate-state friction (6) with the slip-rate field prescribed by the approximate sharp-tip rupture solution (in which the direct effect is neglected compared to the state evolution effect, see main text for details) for various values of $\Delta f_{p} / b$. Deviation of $\Delta f^{r-s}$ from the sharp-tip friction solution $\Delta f / \Delta f_{p}=\exp \left(-\delta / \delta_{c}\right)$ vanishes in the limit of large $\Delta f_{p} / b$. (b) Relative errors of the sharp-tip solution for the fracture energy associated with neglecting the direct effect, (35), and with neglecting variation of the steady-state friction value, (36) with $a / b=1.1$, are shown by solid and dashed lines, respectively. 


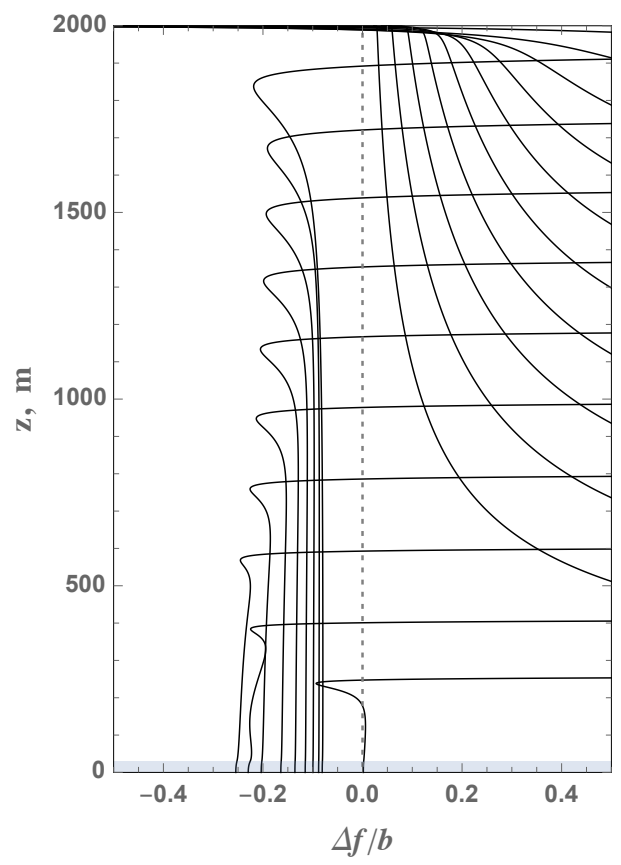

Figure 8: Snapshots of departure of the stress-ratio (friction) from the steady-state, $\Delta f=f-$ $f_{\mathrm{ss}}(V)$, as in Figure 1b, but zoomed in about the steady-state line $(\Delta f=0)$. The relatively small negative $\Delta f \sim 0.1 b$ in the bulk of the propagating crack, compared to the peak values at the rupture front $\sim 10 b$, is the result of the friction direct effect due to the slip deceleration. 